\title{
DIVISOR PROBLEMS AND THE PAIR CORRELATION FOR THE FRACTIONAL PARTS OF $n^{2} \alpha$
}

\author{
JIMI L. TRUELSEN
}

\begin{abstract}
Z. Rudnick and P. Sarnak have proved that the pair correlation for the fractional parts of $n^{2} \alpha$ is Poissonian for almost all $\alpha$. However, they were not able to find a specific $\alpha$ for which it holds. We show that the problem is related to the problem of determining the number of $(a, b, r) \in \mathbf{N}^{3}$ such that $a \leq M, b \leq N, r \leq K$ and $p a b \equiv r(q)$ for $p$ and $q$ coprime. With suitable assumptions on the relative size of $K, M, N$ and $q$ one should expect there to be $K M N / q$ such triples asymptotically and we will show that this holds on average.
\end{abstract}

\section{INTRODUCTION}

For $t \in \mathbf{R}$ and $q \in \mathbf{N}$ let

$$
\|t\|_{q}=\inf _{n \in \mathbf{Z}}|t-q n|,
$$

and set $\|\cdot\|=\|\cdot\|_{1}$. Clearly $\|\cdot\|_{q}$ defines a norm on $\mathbf{R} / q \mathbf{Z}$. For a sequence $\left\{a_{n}\right\}_{1}^{\infty} \subset \mathbf{R} / \mathbf{Z}$, $x>0$ and $N \in \mathbf{N}$ we define

$$
R_{2}\left(x, N,\left\{a_{n}\right\}_{1}^{\infty}\right)=N^{-1} \#\left\{(m, n) \in \mathbf{N}^{2} \mid m, n \leq N, n \neq m,\left\|a_{m}-a_{n}\right\| \leq \frac{x}{N}\right\} .
$$

We say that the pair correlation for $\left\{a_{n}\right\}_{1}^{\infty}$ is Poissonian if for every $x>0$ we have that

$$
\lim _{N \rightarrow \infty} R_{2}\left(x, N,\left\{a_{n}\right\}_{1}^{\infty}\right)=2 x
$$

Note that the limit is not uniform in $x$. We will be particularly interested in the case where $a_{n}$ equals the fractional parts of $n^{2} \alpha$ for $\alpha$ irrational. The spacings between the elements of this sequence correspond to the spacings between the energy levels of the boxed oscillator in quantum mechanics [2]. We define (by an obvious abuse of notation)

$$
R_{2}(x, N, \alpha)=R_{2}\left(x, N,\left\{n^{2} \alpha\right\}_{1}^{\infty}\right)=N^{-1} \#\left\{(m, n) \mid m, n \leq N, n \neq m,\left\|m^{2} \alpha-n^{2} \alpha\right\| \leq \frac{x}{N}\right\} .
$$

Clearly we may as well assume that $0<\alpha<1$. We will be interested in $\alpha$ with certain Diophantine properties. We say that an irrational number $\alpha$ is of type $\kappa$ if

$$
\left|\alpha-\frac{p}{q}\right| \gg \frac{1}{q^{\kappa}}
$$

for all $p \in \mathbf{Z}$ and $q \in \mathbf{N}$. We say that $\alpha$ is "Diophantine" if $\alpha$ is of type $2+\varepsilon$ for any $\varepsilon>0$. In particular all real, irrational algebraic numbers are Diophantine (Roth's theorem - see Theorem 5.7.1 in [10]). Note also that almost all $\alpha$ (with respect to the Lebesgue measure) are Diophantine. To see this we use the identity of sets

$$
\{\beta \in \mathbf{R} \mid \beta \text { is not Diophantine }\}=\bigcup_{n=1}^{\infty} \bigcup_{l \in \mathbf{Z}} \bigcap_{k=1}^{\infty} \bigcup_{q=k}^{\infty} \bigcup_{p=1}^{q}\left[l+\frac{p}{q}-\frac{1}{q^{2+1 / n}}, l+\frac{p}{q}+\frac{1}{q^{2+1 / n}}\right] .
$$

Date: November 21, 2018.

2000 Mathematics Subject Classification. Primary 11B05, 11K31; Secondary 11J54.

The author was supported by a stipend (EliteForsk) from The Danish Agency for Science, Technology and Innovation. 
Let $\mathcal{L}$ denote the Lebesgue measure on the real line. We see that

$$
\mathcal{L}\left(\bigcup_{p=1}^{q}\left[l+\frac{p}{q}-\frac{1}{q^{2+1 / n}}, l+\frac{p}{q}+\frac{1}{q^{2+1 / n}}\right]\right)=2 q^{-(1+1 / n)} .
$$

Since

$$
\sum_{q=1}^{\infty} q^{-(1+1 / n)}<\infty
$$

it follows from the Borel-Cantelli lemma that

$$
\mathcal{L}\left(\bigcap_{k=1}^{\infty} \bigcup_{q=k}^{\infty} \bigcup_{p=1}^{q}\left[l+\frac{p}{q}-\frac{1}{q^{2+1 / n}}, l+\frac{p}{q}+\frac{1}{q^{2+1 / n}}\right]\right)=0 .
$$

Thus the set of non-Diophantine real numbers is a null set.

It is a classical result due to $\mathrm{H}$. Weyl [16] that the sequence $n^{d} \alpha$ is equidistributed modulo 1 for any integer $d \geq 1$. However, it is not true that the pair correlation for the fractional parts of $n^{d} \alpha, d \geq 2$ is Poissonian for all irrational $\alpha$ (for $d=1$ it is never the case - see Exercise 12.6.3 in [10]). A simple construction shows (see [12] p. 62) that $\alpha$ must be at least of type $d+1$.

Z. Rudnick and P. Sarnak have proved [12, Theorem 1] that the pair correlation for the fractional parts of $n^{d} \alpha$ is Poissonian for almost all $\alpha$. Subsequently J. Marklof and A. Strömbergsson [9], and D. R. Heath-Brown [5] have given different proofs in the case $d=2$. However, one does not know of any specific $\alpha$ for which it holds, but Rudnick and Sarnak 12] and Heath-Brown [5] made the following conjecture:

Conjecture 1.1. Assume $\alpha$ is Diophantine. Then the pair correlation for the fractional parts of $n^{2} \alpha$ is Poissonian.

Furthermore, in [5] Heath-Brown was able to show (using a lattice point strategy) that for $\alpha$ of type $9 / 4$

$$
R_{2}(x, N, \alpha)=2 x+O\left(x^{7 / 8}\right),
$$

whenever $1 \leq x \leq \log N$, where the constant implied depends on $\alpha$. This supports Conjecture 1.1 and suggests that perhaps the condition on the Diophantine approximation in the conjecture can be relaxed to some extend.

We remark that the $m$-level correlation for the fractional parts of $n^{2} \alpha$ has been studied by Rudnick, Sarnak and Zaharescu in [13] and by Zaharescu in [17. It is not known if the fractional parts of $n^{2} \alpha$ for almost all $\alpha$ have Poissonian behavior, i.e. have the same distribution as a sequence of independent and uniformly distributed random variables, but it is expected (cf. the conjecture on page 38 in [13]).

In this paper we will only be concerned with Conjecture 1.1 (not higher level correlations). We suggest a line of attack that is based on the study of the function

$$
\tau_{M, N}(m)=\#\left\{(a, b) \in \mathbf{N}^{2} \mid a \leq M, b \leq N, a b=m\right\},
$$

where $m \in \mathbf{N}$ and $M, N \geq 1$. We also define $\tau_{M}^{*}=\tau_{M, M}$. We make the following conjecture:

Conjecture 1.2. Let $K, M, N \geq 1$ with $M \asymp N$ (i.e. $C_{1} N \leq M \leq C_{2} N$ ) and $K \geq N^{\eta}$ for some $\eta>0$. Assume also that $q \leq N^{2-\delta}$ for some $\delta>0$ and $(q, \rho)=1$. Then

$$
\sum_{r \leq K} \sum_{m \equiv \rho r(q)} \tau_{M, N}(m) \sim \frac{K M N}{q}
$$

as $N \rightarrow \infty$ uniformly in $M, K, q$ and $\rho$. The rate of convergence may depend on $\eta, \delta, C_{1}$ and $C_{2}$.

Conjecture 1.2 has applications to the pair correlation problem at hand. We will show that: 
Proposition 1.3. Conjecture 1.2 implies that the pair correlation for the fractional parts of $n^{2} \alpha$ is Poissonian for any $\alpha$ of type $3-\delta$ for any $\delta \in(0,1)$.

This is an immediate consequence of Proposition 2.3. As mentioned previously the pair correlation for the fractional parts of $n^{2} \alpha$ is not Poissonian if $\alpha$ is not of type 3. Conjecture 1.2 claims that $3-\delta$ is sufficient.

Conjecture 1.2 seems bold but natural. Indeed the conjecture provably holds if $q$ is smaller than $N^{1-\delta}$ (see Proposition 3.2 below). However, it turns out that we need $q \geq N^{3 / 2+\delta}$ for our purpose. We can actually obtain partial results for larger $q$ as well based on a lattice point approach using the ideas of Heath-Brown [5]. Before we can state the result we introduce some terminology. We say that a rational number $p / q$ is of type $(e, \mathcal{K})$ if

$$
\left|\frac{p}{q}-\frac{u}{v}\right| \geq \frac{1}{\mathcal{K} v^{e}}
$$

for any rational number $u / v$ with $u / v \neq p / q$. One easily checks that if $\alpha$ is an irrational number of type $e$ then there exists $\mathcal{K}>0$ such that the convergents will be of type $(e, \mathcal{K})$ from some step.

Modifying the proof of (1.1) we prove the following ( $\tau$ denotes the ordinary divisor function):

Theorem 1.4. Let $K, M, N \geq 3$ with $M \asymp N$ and let $\gamma \in(0,1)$. Assume that

$$
q^{1+\delta} \leq\left(\frac{N^{2}}{K}\right)^{1 /(1+\gamma)}
$$

for some $\delta>0$ and $K N / q \geq 1$. Then

$$
\sum_{|r| \leq K} \sum_{p m \equiv r(q)} \tau_{M, N}(m)=\frac{2 K M N}{q}+O\left(N(K N / q)^{7 / 8}+\frac{N^{2}}{q}\left(\tau(q)^{2}(\log N)^{3}+\frac{K(\log \log N)^{2}}{(\log N)^{1 / 4}}\right)\right)
$$

uniformly in $M, K, p$ and $q$ for $p / q$ of type $(2+\gamma, \mathcal{K})$.

It is well known (see e.g. [6]) that one expects that

$$
\sum_{\substack{n \leq x \\ n \equiv r(q)}} \tau(n) \sim \frac{x}{q^{2}} \log x \sum_{d \mid(q, r)} \sum_{c \mid \frac{q}{d}} d c \mu\left(\frac{q}{d c}\right)
$$

as $x \rightarrow \infty$ for $q \leq x^{1-\delta}$ for some $\delta>0$. Average results supporting this conjecture have been considered by Banks, Heath-Brown and Shparlinski [1], and Blomer [3]. If we adapt (1.3) to $\tau_{M, N}$ we should expect that

$$
\sum_{n \equiv r(q)} \tau_{M, N}(n) \sim \frac{M N}{q^{2}} \sum_{d \mid(q, r)} \sum_{c \mid \frac{q}{d}} d c \mu\left(\frac{q}{d c}\right)
$$

for $M \asymp N$ and $q \leq N^{2-\delta}$. Note that

$$
\sum_{d \mid(q, r)} \sum_{c \mid \frac{q}{d}} d c \mu\left(\frac{q}{d c}\right)=\sum_{d \mid(q, r)} d \varphi\left(\frac{q}{d}\right)
$$

It has been proved by Linnik and Vinogradov [8] that

$$
\sum_{\substack{m \leq x \\ m \equiv r(q)}} \tau(m) \ll \frac{\varphi(q) x \log x}{q^{2}}
$$

for $q \leq x^{1-\delta}$ and $(r, q)=1$, where the constant implied depends on $\delta>0$ only. In view of Conjecture 1.2 and (1.6) it would be interesting to find upper bounds for

$$
\sum_{m \equiv r(q)} \tau_{N}^{*}(m)
$$


Heath-Brown [5] suggested the following conjecture which is the analogue of (1.6) for $\tau_{N}^{*}$ :

Conjecture 1.5. Let $\delta \in(0,1)$. Then

$$
\sum_{m \equiv r(q)} \tau_{N}^{*}(m) \ll \frac{\varphi(q) N^{2}}{q^{2}}
$$

uniformly for $(r, q)=1$ and $q \leq N^{2-\delta}$, where the constant implied depends only on $\delta$.

Using the work of M. Nair and G. Tenenbaum [11] we prove an upper bound for the sum in Conjecture 1.5.

Proposition 1.6. Let $q \leq N^{2-\delta}$. Then

$$
\sum_{m \equiv r(q)} \tau_{N}^{*}(m) \ll \frac{N^{2}}{\varphi(q)} e^{\sqrt{(2+\varepsilon)(\log \log N)(\log \log \log N)}}
$$

uniformly for $(r, q)=1$ for any $\varepsilon>0$.

Note that the estimate in the proposition above is off by less than a factor of $(\log N)^{\varepsilon} \operatorname{compared}$ to Conjecture 1.5 since $q / \varphi(q) \ll \log \log q$.

The function $\tau_{M, N}$ is complicated. There is another similar function of interest

$$
\tau_{M}(m)=\#\{d \in \mathbf{N}|d \leq M, d| m\} .
$$

The function $\tau_{M}$ is in many ways simpler than $\tau_{M, N}$. The estimate corresponding to Conjecture 1.5 holds. More precisely we prove:

Theorem 1.7. Let $0<\delta \leq 1,0<\varepsilon<\frac{1}{8}, 0<\kappa$ and $2 \leq N$. Assume also that $N \geq q^{\kappa}$. Then

$$
\sum_{\substack{x<n \leq x+y \\ n \equiv r(q)}} \tau_{N}(n) \ll \frac{y \varphi(q) \log N}{q^{2}}
$$

uniformly for $N,(r, q)=1, x^{\frac{1+4 \varepsilon \delta}{1+\delta}} \leq y \leq x, x \geq c_{0} q^{1+\delta}$, where $c_{0}$ and the constant implied depends at most on $\delta$, $\varepsilon$ and $\kappa$. In particular

$$
\sum_{\substack{m \equiv r(q) \\ m \leq x}} \tau_{N}(m) \ll \frac{\varphi(q) x \log N}{q^{2}} .
$$

Note that with $N=x+y$ we obtain

$$
\sum_{\substack{m \equiv r(q) \\ x<m \leq x+y}} \tau(m) \ll \frac{y \varphi(q) \log x}{q^{2}} .
$$

This extension of (1.6) was also obtained by P. Shiu [14].

Finally we show that Conjecture 1.2 and Conjecture 1.5 hold on average. Indeed we start by proving that (1.4) holds for most values of $q$ and $r$ if $(q, r)$ is small:

Theorem 1.8. Let $\delta>0$ and assume $M \asymp N$. Then

$$
\sum_{(r, q)=k}\left(\sum_{m \equiv r(q)} \tau_{M, N}(m)-\frac{M N}{q^{2}} \sum_{d \mid k} \sum_{c \mid \frac{q}{d}} d c \mu\left(\frac{q}{d c}\right)\right)^{2} \ll \frac{N^{\max (7 / 2,4-\delta)+\varepsilon}}{q}
$$

uniformly for $q \leq N^{2-\delta}$ and $k \mid q$.

From Theorem 1.8 we can deduce the following: 
Theorem 1.9. Let $M, N \geq 1$ with $M \asymp N, q \in \mathbf{N}$ and $K \geq N^{\eta}$ for some $\eta>0$. Assume also that $q \leq N^{2-\delta}$ for some $\delta>0$. Then

$$
\sum_{(\rho, q)=1}\left(\sum_{r \leq K} \sum_{m \equiv \rho r(q)} \tau_{M, N}(m)-\frac{K M N}{q}\right)^{2} \ll \frac{K^{2} N^{4}}{q}\left(q^{\varepsilon}\left(\frac{1}{q}+\frac{1}{K}\right)^{2}+N^{\max (-1 / 2,-\delta)+\varepsilon}\right)
$$

for any $\varepsilon>0$.

In Proposition 3.2 we show that Conjecture 1.2 holds for $q \leq N^{1-\delta}$. Thus we can safely restrict our attention to the case where $q \geq \sqrt{N}$. We have the following corollary, which states that Conjecture 1.2 is true on average:

Corollary 1.10. Let $M, N \geq 1$ with $M \asymp N, q \in \mathbf{N}$ and $K \geq N^{\eta}$ for some $\eta>0$. Assume also that $\sqrt{N} \leq q \leq N^{2-\delta}$ for some $\delta>0$. Then

$$
\frac{1}{\varphi(q)} \sum_{(\rho, q)=1}\left(\frac{q}{K M N} \sum_{r \leq K} \sum_{m \equiv \rho r(q)} \tau_{M, N}(m)-1\right)^{2} \ll N^{-\min (1 / 2, \delta, 2 \eta)+\varepsilon}
$$

for any $\varepsilon>0$.

The author would like to thank P. Sarnak for suggesting the problem of relating Conjecture 1.1 to a divisor problem and D. R. Heath-Brown for generously sharing his ideas on the problem and providing crucial assistance at various stages. The author would also like to thank M. Risager and A. Strömbergsson for comments on an earlier version of the manuscript.

\section{Reducing the Question to an Arithmetic Problem}

Set

$$
S(x, N, \alpha)=\frac{\#\left\{(a, b) \in \mathbf{N} \times \mathbf{Z}|1 \leq a<2 N, 1 \leq| b|\leq N-| N-a|, 2| a+b,\|a b \alpha\| \leq \frac{x}{N}\right\}}{N}
$$

By factoring $m^{2}-n^{2}$ into $a=m+n$ and $b=m-n$ we see that

$$
\begin{aligned}
0 & \leq S(x, N, \alpha)-R_{2}(x, N, \alpha) \\
& \leq \frac{2}{N}+\frac{2}{N} \#\left\{n \in \mathbf{N} \mid n \leq N,\left\|n^{2} \alpha\right\| \leq \frac{x}{N}\right\} \\
& \rightarrow 0
\end{aligned}
$$

as $N \rightarrow \infty$ (the difference between $S$ and $R_{2}$ is that in $S$ we do not exclude all the cases corresponding to $m$ or $n$ equal to 0 ). This follows since the fractional parts of $n^{2} \alpha$ becomes equidistributed in the unit interval. Thus if we want to study Poissonian behavior we may as well study $S(x, N, \alpha)$ rather than $R_{2}(x, N, \alpha)$.

From the elementary theory of continued fractions (see [10] Chapter 7) we know that the convergents $p_{n} / q_{n}$ of $\alpha$ satisfy

$$
\left|\alpha-\frac{p_{n}}{q_{n}}\right| \leq \frac{1}{q_{n} q_{n+1}}
$$

and

$$
q_{n+1} p_{n}-p_{n+1} q_{n}= \pm 1
$$

Define $\mathfrak{R}(y, N, p, q)$ by

$$
\#\left\{(a, b) \in \mathbf{N} \times \mathbf{Z}|1 \leq a<2 N, 1 \leq| b|\leq N-| N-a|, 2| a+b,\|a b p\|_{q} \leq y\right\} .
$$

We have the following: 
Proposition 2.1. Let $\alpha$ be irrational with convergents $p_{n} / q_{n}$. The pair correlation for the fractional parts of $n^{2} \alpha$ is Poissonian if and only if there for all fixed $x>0$ exists $\kappa>0$ and $a$ sequence $\left\{n_{k}\right\}_{1}^{\infty}$ such that $N^{3+\kappa} \ll q_{n_{N}} q_{n_{N}+1}$ and

$$
\mathfrak{R}\left(\frac{x q_{n_{N}}}{N}, N, p_{n_{N}}, q_{n_{N}}\right) \sim 2 x N
$$

as $N \rightarrow \infty$.

Proof. Note that

$$
\|a b \alpha\| \leq \frac{x}{N}
$$

if and only if

$$
\left\|a b p_{n}+a b q_{n}\left(\alpha-\frac{p_{n}}{q_{n}}\right)\right\|_{q_{n}} \leq \frac{x q_{n}}{N} .
$$

Now

$$
\left|a b q_{n}\left(\alpha-\frac{p_{n}}{q_{n}}\right)\right| \leq \frac{N^{2}}{q_{n+1}}
$$

and this implies that

$$
\mathfrak{R}\left(\frac{x q_{n}}{N}-\frac{N^{2}}{q_{n+1}}, N, p_{n}, q_{n}\right) \leq N S(\alpha, N, x) \leq \mathfrak{R}\left(\frac{x q_{n}}{N}+\frac{N^{2}}{q_{n+1}}, N, p_{n}, q_{n}\right) .
$$

Assume $\frac{N^{2}}{q_{n+1}}=o\left(\frac{x q_{n}}{N}\right)$ as $N \rightarrow \infty$. Since $\mathfrak{R}(y, N, p, q)$ is an increasing function of $y$ we conclude that for any $\varepsilon>0$

$$
\mathfrak{R}\left(\frac{(x-\varepsilon) q_{n}}{N}, N, p_{n}, q_{n}\right) \leq N S(\alpha, N, x) \leq \mathfrak{R}\left(\frac{(x+\varepsilon) q_{n}}{N}, N, p_{n}, q_{n}\right)
$$

for $N$ sufficiently large. From this the result follows easily.

Now we have an arithmetic version of Conjecture 1.1. However, the constraints on $a$ and $b$ in the definition of $\mathfrak{R}(y, N, p, q)$ are a bit complicated. We can split $\mathfrak{R}(y, N, p, q)$ into some nicer pieces. The hope is that we can say something about these. This is where Conjecture 1.2 enters the picture as we will see below. First we make a (technical) conjecture:

Conjecture 2.2. Let $\mathcal{K}, \lambda, \zeta, c>0$ be constants with $\lambda<1$. Let $N \geq 1$ and assume $p / q \in \mathbf{Q}$ (with $(p, q)=1)$ is of type $(2+\lambda, \mathcal{K})$ and $N^{\frac{3}{2+\lambda}} \leq q \leq N^{\frac{3(1+\lambda)}{2+\lambda}}$. Then

$$
\sum_{|r| \leq \frac{\zeta q}{N}} \sum_{p m \equiv r(q)} \tau_{c N, N}(m) \sim 2 \zeta c N
$$

as $N \rightarrow \infty$ uniformly in $q$ and $p$.

Clearly Conjecture 1.2 implies Conjecture 2.2. From the next proposition we may therefore conclude that Conjecture 1.2 implies Conjecture 1.1.

Proposition 2.3. Assume Conjecture 2.2 holds with some $\lambda \in(0,1)$ and for any $\mathcal{K}>1$. For $\alpha$ of type $2+\beta$ with $\beta<\lambda$ the pair correlation for the fractional parts of $n^{2} \alpha$ is Poissonian. In particular Conjecture 1.1 holds.

Proof. Let us first ignore the technical conditions on $N, p$ and $q$. We see that

$$
\begin{aligned}
& \mathfrak{R}\left(\frac{x q}{N}, N, p, q\right)=\#\left\{(a, b) \in \mathbf{N}^{2}|a, b \leq N, 2| a+b,\|a b p\|_{q} \leq x q / N\right\}+ \\
& 2 \#\left\{(a, b) \in \mathbf{N}^{2}|N<a<2 N, 0<b \leq 2 N-a, 2| a+b,\|a b p\|_{q} \leq x q / N\right\}+ \\
& \quad \#\left\{a \in \mathbf{N} \mid a \leq N,\left\|a^{2} p\right\|_{q} \leq x q / N\right\} .
\end{aligned}
$$


The last term is negligible as $N \rightarrow \infty$, since the sequence $\left\{n^{2} \alpha\right\}_{1}^{\infty}$ is equidistributed modulo 1 . We will see that the first two terms are of the same size (if we assume Conjecture 2.2). We start by considering the first term. We define

$$
\mathfrak{T}_{M}(m)=\#\left\{(a, b) \in \mathbf{N}^{2} \mid a, b \leq M, a \equiv b(2), a b=m\right\} .
$$

Note that

$$
\mathfrak{T}_{M}(m)= \begin{cases}\tau_{M}^{*}(m) & \text { if } m \equiv 1(2) \\ 0 & \text { if } m \equiv 2(4) \\ \tau_{M / 2}^{*}(m / 4) & \text { if } m \equiv 0(4)\end{cases}
$$

Using this notation we see that (assuming that $N>2 x$ )

$$
\#\left\{(a, b) \in \mathbf{N}^{2}|a, b \leq N, 2| a+b,\|a b p\|_{q} \leq x q / N\right\}=\sum_{|r| \leq \frac{x q}{N}} \sum_{m \equiv r \bar{p}(q)} \mathfrak{T}_{N}(m),
$$

where $\bar{p}$ is the inverse of $p$ modulo $q$. Using (2.4) we can write this as

$$
\sum_{|r| \leq \frac{x q}{N}} \sum_{m \equiv r \bar{p}(q)} \mathfrak{T}_{N}(m)=\sum_{|r| \leq \frac{x q}{N}}\left(\sum_{2 k+1 \equiv r \bar{p}(q)} \tau_{N}^{*}(2 k+1)+\sum_{4 l \equiv r \bar{p}(q)} \tau_{N / 2}^{*}(l)\right) .
$$

Furthermore we see that

$$
\sum_{|r| \leq \frac{x q}{N}} \sum_{4 l \equiv r \bar{p}(q)} \tau_{N / 2}^{*}(l)=\left\{\begin{array}{ll}
\sum_{|r| \leq \frac{x q}{N}} \sum_{l \equiv r \overline{4 p}(q)} \tau_{N / 2}^{*}(l) & \text { if } q \equiv 1(2) \\
\sum_{|r| \leq \frac{x q}{2 N}} \sum_{l \equiv r \overline{2 p}(q / 2)} \tau_{N / 2}^{*}(l) & \text { if } 2 \| q \\
\sum_{|r| \leq \frac{x q}{4 N}} \sum_{l \equiv r \bar{p}(q / 4)} \tau_{N / 2}^{*}(l) & \text { if } 4 \mid q
\end{array} .\right.
$$

By (2.5) Conjecture 2.2 implies that

$$
\sum_{|r| \leq \frac{x q}{N}} \sum_{4 l \equiv r \bar{p}(q)} \tau_{N / 2}^{*}(l) \sim \frac{1}{2} x N .
$$

Note also that

$$
\sum_{2 k+1 \equiv r \bar{p}(q)} \tau_{N}^{*}(2 k+1)=\sum_{m \equiv r \bar{p}(q)} \tau_{N}^{*}(m)-\sum_{2 m \equiv r \bar{p}(q)} \tau_{N}^{*}(2 m) .
$$

If $q$ is odd then

$$
\sum_{2 m \equiv r \bar{p}(q)} \tau_{N}^{*}(2 m)=2 \sum_{m \equiv \overline{\overline{2 p}}(q)} \tau_{N, N / 2}(m)-\sum_{m \equiv r \overline{4}(q)} \tau_{N / 2}^{*}(m) .
$$

Thus Conjecture 2.2 implies

$$
\sum_{|r| \leq \frac{x q}{N}} \sum_{2 k+1 \equiv r \bar{p}(q)} \tau_{N}^{*}(2 k+1) \sim \frac{1}{2} x N .
$$

Now assume $q$ is even. This implies that $p$ and $\bar{p}$ are odd. Thus $m$ is even if and only if $r$ is even. Hence

$$
\begin{aligned}
\sum_{|r| \leq \frac{x q}{N}} \sum_{2 m \equiv r \bar{p}(q)} \tau_{N}^{*}(2 m)= & 2 \sum_{|r| \leq \frac{x q}{2 N}} \sum_{m \equiv r \bar{p}(q / 2)} \tau_{N, N / 2}(m)- \\
& \begin{cases}\sum_{|r| \leq \frac{x q}{2 N}} \sum_{m \equiv r} \overline{2 p}(q / 2) & \tau_{N / 2}^{*}(m) \text { if } 2 \| q \\
\sum_{|r| \leq \frac{x q}{4 N}} \sum_{m \equiv r \bar{p}(q / 4)} \tau_{N / 2}^{*}(m) & \text { if } 4 \mid q \\
\sim & \frac{3}{2} x N .\end{cases}
\end{aligned}
$$


Thus we conclude that

$$
\sum_{|r| \leq \frac{x q}{N}} \sum_{m \equiv r \bar{p}(q)} \mathfrak{T}_{N}(m) \sim x N
$$

Now we consider the second term in (2.3) and we set

$$
\mathfrak{S}=\#\left\{(a, b) \in \mathbf{N}^{2}|N<a<2 N, 0<b \leq 2 N-a, 2| a+b,\|a b p\|_{q} \leq x q / N\right\} .
$$

For $k, l_{1}, l_{2} \in \mathbf{N}$ such that $l_{1}+l_{2} \leq 2^{k}$ we define

$$
T_{l_{1}, l_{2}}(k)=\#\left\{(a, b) \in \mathbf{N}^{2}\left|a \in I\left(k, l_{1}\right), b \in J\left(k, l_{2}\right), 2\right| a+b,\|a b p\|_{q} \leq x q / N\right\},
$$

where

$$
I\left(k, l_{1}\right)=\left(N\left(1+\left(l_{1}-1\right) / 2^{k}\right), N\left(1+l_{1} / 2^{k}\right)\right]
$$

and

$$
J\left(k, l_{2}\right)=\left(N\left(l_{2}-1\right) / 2^{k}, N l_{2} / 2^{k}\right] .
$$

As before we deduce (using Conjecture 2.2) that

$$
T_{l_{1}, l_{2}}(k) \sim \frac{1}{4^{k}} x N
$$

uniformly in $l_{1}$ and $l_{2}$ (since $k$ is fixed). Clearly we have

$$
\begin{aligned}
\sum_{\substack{l_{1}, l_{2} \\
l_{1}+l_{2} \leq 2^{k}-1}} & T_{l_{1}, l_{2}}(k) \\
& \leq \#\left\{(a, b) \in \mathbf{N}^{2}|N<a<2 N, 0<b \leq 2 N-a, 2| a+b,\|a b p\|_{q} \leq x q / N\right\} \\
& \leq \sum_{\substack{l_{1}, l_{2} \\
l_{1}+l_{2} \leq 2^{k}}} T_{l_{1}, l_{2}}(k) .
\end{aligned}
$$

Recall that \#\{(l, $\left.\left.l_{2}\right) \in \mathbf{N}^{2} \mid l_{1}+l_{2} \leq m\right\}=\frac{m(m+1)}{2}$. Using (2.6) we see that

$$
\frac{1-2^{-k}}{2} x \leq \liminf _{N} \frac{\mathfrak{S}}{N} \leq \limsup _{N} \frac{\mathfrak{S}}{N} \leq \frac{1+2^{-k}}{2} x
$$

Since this holds for any $k$ we must have $\mathfrak{S} \sim x N / 2$ as desired. By Proposition 2.1 it remains to prove that there exists $\kappa>0$ such that for each $N$ sufficiently large we can choose $q_{n}$ and $q_{n+1}$ such that $N^{3+\kappa} \ll q_{n} q_{n+1}$. By Conjecture 2.2 we must take $q_{n} \leq N^{\frac{3(1+\lambda)}{(2+\lambda)}}$. Recall that $\alpha$ is of type $2+\beta$. Choose $n$ such that

$$
q_{n} \leq N^{\frac{3(1+\lambda)}{(2+\lambda)}} \leq q_{n+1} .
$$

The condition that $\alpha$ is of type $2+\beta$ implies that

$$
q_{n} q_{n+1} \ll q_{n}^{2+\beta}
$$

and hence

$$
q_{n} q_{n+1} \gg q_{n+1}^{1+\frac{1}{1+\beta}} \geq N^{\frac{3(1+\lambda)}{(2+\lambda)}\left(1+\frac{1}{1+\beta}\right)} .
$$

Thus we can choose $\kappa=\frac{\lambda-\beta}{(1+\beta)(2+\lambda)}$.

It should be mentioned that there may be some loss in using the rational approximation at an early stage in Proposition 2.1. For this approach to work we must be able to work with $q \geq N^{3 / 2+\delta}$. In Theorem 1.4 we can say something about values of $q$ that are slightly smaller. In the proof of (1.1) Heath-Brown was able to work with $\alpha$ rather than its convergents and only use the Diophantine approximation at the very end of the proof allowing the use of smaller values of $q$. 
By condition (2.2) we see that the inverse of $p_{n}$ modulo $q_{n}$ is $\pm q_{n+1}$. To begin with one could study

$$
\sum_{|r| \leq \frac{x q_{n}}{N}} \sum_{m \equiv r q_{n+1}\left(q_{n}\right)} \tau_{N}^{*}(m)
$$

Perhaps one can use this information to say more about the pair correlation problem for specific $\alpha$ 's such as $\sqrt{2}$ or the golden ratio where the $q_{n}$ 's are known.

\section{Preliminary Evidence for Conjecture 1.2}

We will now explain why we should expect the asymptotics in Conjecture 1.2. We try the "naive" approach. Define

$$
\delta_{d}(n)=\left\{\begin{array}{ll}
1 & \text { if } d \mid n \\
0 & \text { if } d \nmid n
\end{array} .\right.
$$

Assume $M, N \geq 2$. Clearly $\tau_{M, N}(m)=\sum_{d=\lceil m / N\rceil}^{M} \delta_{d}(m)$. Thus

$$
\begin{aligned}
\sum_{r \leq K} \sum_{m \equiv \rho r(q)} \tau_{M, N}(m) & =\sum_{r \leq K} \sum_{l \leq\left(M N-[r \rho]_{q}\right) / q} \sum_{d=\left[\left(q l+[r \rho]_{q}\right) / N\right\rceil}^{M} \sum_{r \leq K} \sum_{l \leq\left(M N-[r \rho]_{q}\right) / q} \sum_{\left(q l+[r \rho]_{q}\right) / N \leq d \leq M} \delta_{d}\left(q l+[r \rho]_{q}\right) \\
& =\sum_{r \leq K} \sum_{[r \rho]_{q} / N \leq d \leq M} \delta_{d \leq l \leq\left(N d-[r \rho]_{q}\right) / q}\left(q l+[r \rho]_{q}\right)+O\left(N^{2} q^{-1+\varepsilon}\right)
\end{aligned}
$$

where $[\cdot]_{q}$ denotes the remainder when dividing by $q$. Now the length of the $l$-interval can be much smaller than $d$ and this is where the approach fails. We should expect that

$$
\sum_{[r \rho]_{q} / N \leq d \leq M} \sum_{0 \leq l \leq\left(N d-[r \rho]_{q}\right) / q} \delta_{d}\left(q l+[r \rho]_{q}\right)
$$

is heuristically

$$
\sum_{\substack{[r \rho]_{q} / N \leq d \leq M \\(d, q) \mid r}} \frac{N d-[r \rho]_{q}}{d q}(d, q)=\frac{N}{q} \sum_{\substack{d \leq M \\(d, q) \mid r}}(d, q)+O\left(\sum_{\substack{d \leq M \\(d, q) \mid r}} \frac{(d, q)}{d}\right)+O\left(\frac{N}{q} \sum_{\substack{d \leq q / N \\(d, q) \mid r}}(d, q)\right)
$$

and it is the case if $q \leq N^{1-\delta}$. Using Lemma 3.1 below we see that the "expected" value of $\sum_{r \leq K} \sum_{m \equiv \rho r(q)} \tau_{M, N}(m)$ is

$$
\frac{K M N}{q}+O\left((K+N) N q^{-1+\varepsilon}+K q^{\varepsilon}\right) .
$$

On numerous occasions we will use the fact that

$$
\varphi(x, q)=\#\{n \in \mathbf{N} \mid n \leq x,(n, q)=1\}=\sum_{d \mid q} \mu(d)\left[\frac{x}{d}\right]=\frac{\varphi(q) x}{q}+O(\tau(q)) .
$$

This implies that for $k \mid q$

$$
\#\{r \in \mathbf{N} \mid r \leq K,(r, q)=k\}=\frac{K \varphi(q / k)}{q}+O\left(q^{\varepsilon}\right) .
$$


Lemma 3.1. Let $N, K \geq 1$ and $q \in \mathbf{N}$. Then

$$
\begin{gathered}
\sum_{r \leq K} \sum_{\substack{d \leq N \\
(d, q) \mid r}}(d, q)=K N+O\left((K+N) q^{\varepsilon}\right), \\
\sum_{r \leq K} \sum_{\substack{d \leq N \\
(d, q) \mid r}} \frac{(d, q)}{d}=K \log N+O\left(K q^{\varepsilon}\right)+O\left(q^{\varepsilon} \log N\right)
\end{gathered}
$$

$$
\sum_{r \leq K} r \sum_{\substack{d \leq N \\(d, q) \mid r}}(d, q)=\frac{1}{2} N K^{2}+O\left(K(K+N) q^{\varepsilon}\right)
$$

for any $\varepsilon>0$.

Proof. We see that

$$
\begin{aligned}
\sum_{r \leq K} \sum_{\substack{d \leq N \\
(d, q) \mid r}}(d, q)= & \sum_{\substack{k \mid q \\
k \leq K}} k \#\left\{(r, d) \in \mathbf{N}^{2}|r \leq K, d \leq N,(d, q)=k, k| r\right\} \\
= & \sum_{\substack{k \mid q \\
k \leq K}} k\left[\frac{K}{k}\right] \#\{d \in \mathbf{N} \mid d \leq N,(d, q)=k\} \\
= & \sum_{\substack{k \mid q \\
k \leq K}} k\left[\frac{K}{k}\right] \sum_{a \mid q / k} \mu(a)\left[\frac{N}{a k}\right] \\
= & \sum_{\substack{k \mid q \\
k \leq K}} k\left(\frac{K}{k}+O(1)\right) \sum_{a \mid q / k} \mu(a)\left(\frac{N}{a k}+O(1)\right) \\
= & K N \sum_{\substack{k \mid q \\
k \leq K}} \sum_{a \mid q / k} \frac{\mu(a)}{a k}+O\left(K q^{\varepsilon}\right)+O\left(N q^{\varepsilon}\right)
\end{aligned}
$$

for any $\varepsilon>0$. Now

$$
\sum_{\substack{k \mid q \\ k \leq K}} \sum_{a \mid q / k} \frac{\mu(a)}{a k}=q^{-1} \sum_{\substack{k \mid q \\ k \leq K}} \varphi(q / k)=1-q^{-1} \sum_{\substack{k \mid q \\ k>K}} \varphi(q / k)
$$

and

$$
\sum_{\substack{k \mid q \\ k>K}} \varphi(q / k) \leq q \sum_{\substack{k \mid q \\ k>K}} \frac{1}{k} \leq \frac{q}{K} \sum_{\substack{k \mid q \\ k>K}} 1=O\left(\frac{q^{1+\varepsilon}}{K}\right)
$$

Thus

$$
\sum_{r \leq K} \sum_{\substack{d \leq N \\(d, q) \mid r}}(d, q)=K N\left(1+O\left(\frac{q^{\varepsilon}}{K}\right)\right)+O\left(K q^{\varepsilon}\right)+O\left(N q^{\varepsilon}\right)=K N+O\left((K+N) q^{\varepsilon}\right) .
$$


Using partial summation we see that

$$
\begin{aligned}
\sum_{r \leq K} \sum_{\substack{d \leq N \\
(d, q) \mid r}} \frac{(d, q)}{d} & =N^{-1} \sum_{r \leq K} \sum_{\substack{d \leq N \\
(d, q) \mid r}}(d, q)+\int_{1}^{N} \frac{1}{t^{2}} \sum_{r \leq K} \sum_{\substack{d \leq t \\
(d, q) \mid r}}(d, q) d t \\
& =K+O\left((K / N+1) q^{\varepsilon}\right)+K \log N+O\left(K q^{\varepsilon}\right)+O\left(q^{\varepsilon} \log N\right) \\
& =K \log N+O\left((K+1) q^{\varepsilon}\right)+O\left(q^{\varepsilon} \log N\right) .
\end{aligned}
$$

The last part of the proposition is also proved using partial summation. We omit the details.

The difficulty in proving Conjecture 1.2 obviously lies in dealing with the fact that we only consider a very small number of all the residue classes ( $K$ can be much smaller than $q$ ) and at the same time $M$ and $N$ can be much smaller than $q$ (as pointed out earlier). Indeed the above shows:

Proposition 3.2. Let $K, M, N \geq 1$ with $M \asymp N$ and $K \geq N^{\eta}$ for some fixed $\eta>0$. Assume also that $q \leq N^{1-\delta}$ for some $\delta>0$ and $(q, \rho)=1$. Then

$$
\sum_{r \leq K} \sum_{m \equiv \rho r(q)} \tau_{M, N}(m) \sim \frac{K M N}{q}
$$

as $N \rightarrow \infty$ uniformly in $M, K, q$ and $\rho$.

Conjecture 1.2 says that the asymptotic formula above still holds if we extend the range of $q$ to $q \leq N^{2-\delta}$. In the same way we see that Conjecture 1.5 holds for small values of $q$. More precisely we have

$$
\sum_{m \equiv r(q)} \tau_{N}^{*}(m) \ll \frac{N^{2} \varphi(q)}{q^{2}}
$$

for $q \leq N^{1-\delta},(r, q)=1$.

The following lemma will be useful in the next section. It shows that (1.4) would imply the asymptotics in Conjecture 2.2 .

Lemma 3.3. Let $q \in \mathbf{N}$. Then

$$
\sum_{r=1}^{q} \sum_{d \mid(q, r)} \sum_{c \mid \frac{q}{d}} d c \mu\left(\frac{q}{d c}\right)=q^{2}
$$

If $K>0$ then for any $\varepsilon>0$

$$
\sum_{r \leq K} \sum_{d \mid(q, r)} \sum_{c \mid q} d c \mu\left(\frac{q}{d c}\right)=K q+O\left((K+q) q^{\varepsilon}\right) .
$$

Proof. We see that

$$
\sum_{r=1}^{q} \sum_{d \mid(q, r)} \sum_{c \mid \frac{q}{d}} d c \mu\left(\frac{q}{d c}\right)=\sum_{k \mid q} \varphi(q / k) \sum_{d \mid k} \sum_{c \mid \frac{q}{d}} d c \mu\left(\frac{q}{d c}\right) .
$$

Since the left hand side is a multiplicative function of $q$ it suffices to prove that

$$
\sum_{n=0}^{l} \varphi\left(p^{l-n}\right) \sum_{m=0}^{n} \sum_{j=0}^{l-m} p^{m+j} \mu\left(p^{l-m-j}\right)=p^{2 l} .
$$


for a prime $p$. One easily checks that

$$
\begin{aligned}
\sum_{n=0}^{l} \varphi\left(p^{l-n}\right) \sum_{m=0}^{n} \sum_{j=0}^{l-m} p^{m+j} \mu\left(p^{l-m-j}\right) & =p^{l-1}+p^{l}\left(1-p^{-1}\right) \sum_{n=0}^{l} \varphi\left(p^{l-n}\right)(n+1) \\
& =p^{l-1}+p^{l}\left(1-p^{-1}\right)\left(l+1+p^{l}\left(1-p^{-1}\right) \sum_{n=0}^{l-1} \frac{n+1}{p^{n}}\right) .
\end{aligned}
$$

The identity (3.6) now follows since

$$
\sum_{n=0}^{l-1} \frac{n+1}{p^{n}}=\frac{1-p^{-l-1}}{\left(1-p^{-1}\right)^{2}}-\frac{l+1}{p^{l}\left(1-p^{-1}\right)} .
$$

From the first part we deduce using (3.2) that

$$
\begin{aligned}
\sum_{r \leq K} \sum_{d \mid(q, r)} \sum_{c \mid \frac{q}{d}} d c \mu\left(\frac{q}{d c}\right) & =\frac{K}{q} \sum_{\substack{k \mid q \\
k \leq K}} \varphi\left(\frac{q}{k}\right) \sum_{d \mid k} \sum_{c \mid \frac{q}{d}} d c \mu\left(\frac{q}{d c}\right)+O\left(q^{1+\varepsilon}\right) \\
& =K q-\frac{K}{q} \sum_{\substack{k \mid q \\
k>K}} \varphi\left(\frac{q}{k}\right) \sum_{d \mid k} d \varphi\left(\frac{q}{d}\right)+O\left(q^{1+\varepsilon}\right) \\
& =K q+O\left(q^{1+\varepsilon}\right) .
\end{aligned}
$$

\section{Average Results}

From [4] we know how to count elements of an arithmetic progression in a given interval:

Lemma 4.1. Let $a<b, r \in \mathbf{Z}$ and $H \in \mathbf{N}$ with $H<q$. Then

$$
\sum_{\substack{a<m \leq b \\ m \equiv r(q)}} 1=\frac{b-a}{q}+\sum_{1 \leq|h| \leq H} C_{a, b}(h) e(-h r / q)+O\left(\theta_{H}((a-r) / q)+\theta_{H}((b-r) / q)\right),
$$

where

$$
\theta_{H}(s)= \begin{cases}\min (1,1 /(H\|s\|)) & \text { if } s \notin \mathbf{Z} \\ 1 & \text { if } s \in \mathbf{Z}\end{cases}
$$

and

$$
C_{a, b}(n)=\frac{e(b n / q)-e(a n / q)}{2 \pi i n} .
$$

Note that $H_{1} \leq H_{2}$ implies that $\theta_{H_{1}}(s) \geq \theta_{H_{2}}(s)$. It will be convenient to set

$$
E_{H}(a, b, r, q)=\theta_{H}((a-r) / q)+\theta_{H}((b-r) / q) .
$$

In [1] the following lemma was proved:

Lemma 4.2. Let $(r, q)=1, H$ as in Lemma 4.1 and $t \in \mathbf{R}$. Then

$$
\sum_{c \in \mathbf{Z}_{q}} \theta_{H}((t-r c) / q) \ll \frac{q^{1+\varepsilon}}{H}
$$

for any $\varepsilon>0$, where the constant implied depends at most on $\varepsilon$. 
We will use these results to prove a technical lemma. Define

$$
I(m, M, \alpha, \beta, b, t)=\{x \in \mathbf{N} \mid \max (m,(\alpha+t) / b) \leq x \leq \min (M,(\beta+t) / b)\}
$$

and

$$
J(m, M, \alpha, \beta, b, t)=\{x \in \mathbf{N} \mid \max (m,(\alpha+t) / b)<x \leq \min (M,(\beta+t) / b)\} .
$$

Lemma 4.3. Let $N \geq 1$. Then

$$
\begin{aligned}
& \sum_{\substack{a \leq N_{1} \\
b \leq N_{2}}} \sum_{c \in \mathbf{Z}} \#\left\{x \in I\left(1, N_{3}, a, N_{4} a, b, r+c q\right) \mid x b \equiv r+c q(a)\right\} \\
& \quad=\sum_{\substack{a \leq N_{1} \\
b \leq N_{2}}} \sum_{\substack{c \in \mathbf{Z} \\
(a, b) \mid c q+r}} \frac{(a, b)}{a} \int_{0}^{N_{3}} 1_{\left\{t \in \mathbf{R} \mid(r+c q) / b \leq t \leq\left(N_{4} a+r+c q\right) / b\right\}}(t) d t+O\left(\frac{N^{7 / 2+\varepsilon}}{q}\right)
\end{aligned}
$$

for $N_{i} \asymp N, q \leq N^{2-\delta}$ and $|r| \ll N^{4}$. In the first sum $a, b \in \mathbf{N}$.

Proof. We see that

$$
\begin{aligned}
& \sum_{\substack{a \leq N_{1} \\
b \leq N_{2}}} \sum_{c \in \mathbf{Z}} \#\left\{x \in I\left(1, N_{3}, a, N_{4} a, b, r+c q\right) \mid x b \equiv r+c q(a)\right\} \\
& \quad=\sum_{\substack{a \leq N_{1} \\
b \leq N_{2}}} \sum_{c \in \mathbf{Z}} \#\left\{x \in J\left(0, N_{3}, 0, N_{4} a, b, r+c q\right) \mid x b \equiv r+c q(a)\right\} .
\end{aligned}
$$

Note that we can assume that $c \in \widetilde{I}=\left[-\left(M^{2}+r\right) / q,\left(M^{2}-r\right) / q\right]$ since the sum is zero otherwise. Note that the length of $\widetilde{I}$ is $2 M^{2} / q$. We now apply Lemma 4.1 and get

$$
\begin{aligned}
& \sum_{\substack{a \leq N_{1} \\
b \leq N_{2}}} \sum_{c \in \mathbf{Z}} \#\left\{x \in J\left(0, N_{3}, 0, N_{4} a, b, r+c q\right) \mid x b \equiv r+c q(a)\right\} \\
& \quad=\sum_{\substack{a \leq N_{1} \\
b \leq N_{2}}} \sum_{\substack{c \in \mathbf{Z} \\
(a, b) \mid c q+r}} \frac{(a, b)}{a} \int_{0}^{N_{3}} 1_{\left\{t \in \mathbf{R} \mid(r+c q) / b \leq t \leq\left(N_{4} a+r+c q\right) / b\right\}}(t) d t+O\left(\mathcal{F}+\mathcal{E}_{1}+\mathcal{E}_{2}\right)
\end{aligned}
$$

where the error term $\mathcal{E}_{1}$ accounts for the contribution from $E_{H}$ (we choose $H=\left[N^{\lambda}\right]-1$ ) for $a$, $b$ and $c$ such that

$$
\min \left(\frac{a}{(a, r+c q)}, \frac{b}{(b, r+c q)}\right)>N^{\lambda}
$$

$\mathcal{F}$ is the exponential sum (we set $M=\max N_{i}$ )

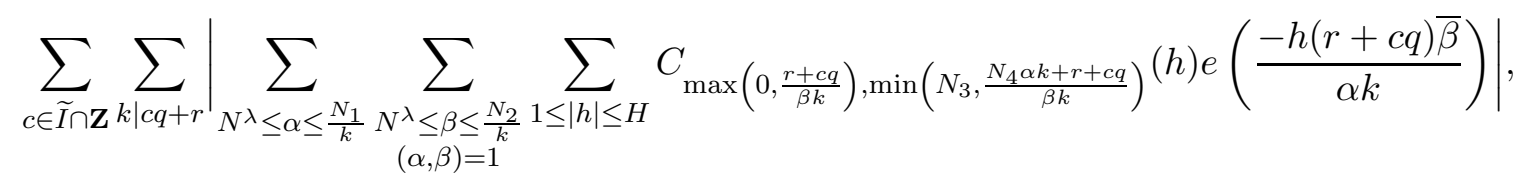

and $\mathcal{E}_{2}$ accounts for the the error coming from $a, b$ and $c$ not satisfying (4.1) and the exponential sum terms with

$$
\frac{\min (a, b)}{(a, b)}<N^{\lambda}
$$

In particular these $a, b$ and $c$ satisfy

$$
\min \left(\frac{a}{(a, r+c q)}, \frac{b}{(b, r+c q)}\right)<N^{\lambda}
$$


Thus $\mathcal{E}_{2}$ is estimated by

$$
\begin{aligned}
\mathcal{E}_{2} & \ll N^{\varepsilon} \sum_{c \in \widetilde{I} \cap \mathbf{Z}} \sum_{k \mid r+c q} \\
& \#\left\{(a, b) \in \mathbf{N}^{2} \mid(a, b)=k, a \leq N_{1}, b \leq N_{2}, \min \left(\frac{a}{(a, r+c q)}, \frac{a}{(a, r+c q)}\right)<N^{\lambda}\right\} \\
& \ll N^{\varepsilon} \sum_{c \in \widetilde{I} \cap \mathbf{Z}} \sum_{k \mid r+c q}\left(\frac{N_{2}}{k} \sum_{\delta_{1} \mid \frac{r+c q}{k}} \#\left\{\alpha \in \mathbf{N} \mid \alpha \leq N^{\lambda} \delta_{1},(\alpha,(r+c q) / k)=\delta_{1}\right\}\right. \\
& \left.+\frac{N_{1}}{k} \sum_{\delta_{2} \mid \frac{r+c q}{k}} \#\left\{\beta \in \mathbf{N} \mid \beta \leq N^{\lambda} \delta_{2},(\beta,(r+c q) / k)=\delta_{2}\right\}\right) \\
& \ll \frac{N^{3+\lambda+2 \varepsilon}}{q} .
\end{aligned}
$$

We see that the error term $\mathcal{E}_{1}$ is at most of order

$$
\sum_{c \in \tilde{I} \cap \mathbf{Z}} \sum_{k \mid c q+r} \sum_{\delta_{1}, \delta_{2} \mid \frac{r+c q}{k}} \sum_{(\alpha, \beta) \in \mathcal{A}} E_{H}\left(\max \left(0, \frac{r+c q}{\beta k}\right), \min \left(N_{3}, \frac{N_{4} \alpha k+r+c q}{\beta k}\right), \frac{\bar{\beta}(r+c q)}{k}, \alpha\right),
$$

where

$$
\mathcal{A}=\left\{(\alpha, \beta) \in \mathbf{N}^{2} \mid \alpha \in I_{1}, \beta \in I_{2},(\alpha, \beta)=1,\left(\alpha, \frac{r+c q}{k}\right)=\delta_{1},\left(\beta, \frac{r+c q}{k}\right)=\delta_{2}\right\},
$$

$I_{1}=\left[\delta_{1} N^{\lambda}, N_{1} / k\right]$ and $I_{2}=\left[\delta_{2} N^{\lambda}, N_{2} / k\right]$. First we consider

$$
\sum_{(\alpha, \beta) \in \mathcal{A}} \theta_{H}\left(\frac{\bar{\beta}(r+c q)}{\alpha k}\right)
$$

From Lemma 4.2 it follows that

$$
\sum_{\substack{\beta \in I_{2} \\(\alpha, \beta) \in \mathcal{A}}} \theta_{H}\left(\frac{\bar{\beta}(r+c q)}{\alpha k}\right) \ll N^{-\lambda}\left(\frac{N_{2}\left(\alpha, \frac{r+c q}{k}\right)}{\alpha k}+1\right)\left(\frac{\alpha}{\left(\alpha, \frac{r+c q}{k}\right)}\right)^{1+\varepsilon}
$$

and

$$
\sum_{\alpha \leq \frac{N_{1}}{k}}\left(\frac{N_{2}\left(\alpha, \frac{r+c q}{k}\right)}{\alpha k}+1\right)\left(\frac{\alpha}{\left(\alpha, \frac{r+c q}{k}\right)}\right)^{1+\varepsilon} \ll N^{2+2 \varepsilon}
$$

Thus it follows that

$$
\sum_{c \in \widetilde{I} \cap \mathbf{Z}} \sum_{k \mid c q+r} \sum_{\delta_{1}, \delta_{2} \mid \frac{r+c q}{k}} \sum_{(\alpha, \beta) \in \mathcal{A}} \theta_{H}\left(\frac{\bar{\beta}(r+c q)}{\alpha k}\right) \ll \frac{N^{4-\lambda+3 \varepsilon}}{q} .
$$

We now consider

$$
\sum_{(\alpha, \beta) \in \mathcal{A}} \theta_{H}\left(\frac{(\bar{\beta}-1 / \beta)(r+c q)}{\alpha k}\right)=\sum_{(\alpha, \beta) \in \mathcal{A}} \theta_{H}\left(\frac{\bar{\alpha}(r+c q)}{\beta k}\right),
$$

where $\bar{\alpha}$ is the inverse of $\alpha$ modulo $\beta$. Again we apply Lemma 4.2 and obtain

$$
\sum_{c \in \widetilde{I} \cap \mathbf{Z}} \sum_{k \mid c q+r} \sum_{(\alpha, \beta) \in \mathcal{A}} \theta_{H}\left(\frac{(\bar{\beta}-1 / \beta)(r+c q)}{\alpha k}\right) \ll \frac{N^{4-\lambda+\varepsilon}}{q} .
$$

The remaining terms of similar type are estimated in the same way. 
We now consider the exponential sum

$$
\sum_{N^{\lambda}<\alpha \leq \frac{N_{1}}{k}} \sum_{\begin{array}{c}
N^{\lambda}<\beta \leq \frac{N_{2}}{k} \\
(\alpha, \beta)=1
\end{array}} \sum_{1 \leq|h| \leq H} C_{\max \left(0, \frac{r+c q}{\beta k}\right), \min \left(N_{3}, \frac{N_{4} \alpha k+r+c q}{\beta k}\right)}(h) e\left(\frac{-h(r+c q) \bar{\beta}}{\alpha k}\right) .
$$

First we look at

$$
\sum_{1 \leq|h| \leq H} \frac{1}{2 \pi i h} \sum_{N^{\lambda}<\alpha \leq \frac{N_{1}}{k}} \sum_{\substack{N^{\lambda}<\beta \leq \frac{N_{2}}{k} \\(\alpha, \beta)=1}} e\left(\frac{-h(r+c q) \bar{\beta}}{\alpha k}\right) .
$$

Using standard exponential sum techniques we rewrite the inner sum (see e.g. [1])

$$
\begin{aligned}
\sum_{\substack{N^{\lambda}<\beta \leq \frac{N_{2}}{k} \\
(\alpha, \beta)=1}} e\left(\frac{-h(r+c q) \bar{\beta}}{\alpha k}\right) & =\sum_{\substack{\beta=0 \\
(\alpha, \beta)=1}}^{\alpha-1} e\left(\frac{-h(r+c q) \bar{\beta}}{\alpha k}\right) \sum_{N^{\lambda}<\zeta \leq \frac{N_{2}}{k}} \frac{1}{\alpha} \sum_{\xi=-\left\lfloor\frac{\alpha-1}{2}\right\rfloor}^{\left\lfloor\frac{\alpha}{2}\right\rfloor} e\left(\frac{\xi(\beta-\zeta)}{\alpha}\right) \\
& =\frac{1}{\alpha} \sum_{\xi=-\left\lfloor\frac{\alpha-1}{2}\right\rfloor} \sum_{\substack{\beta=0 \\
(\alpha, \beta)=1}}^{\alpha-1} e\left(\frac{\xi \beta-h \bar{\beta}(r+c q) / k}{\alpha}\right) \sum_{N^{\lambda}<\zeta \leq \frac{N_{2}}{k}} e\left(-\frac{\xi \zeta}{\alpha}\right) .
\end{aligned}
$$

The last sum is just a Weyl sum and is easily estimated (see e.g. [7] Section 8.2) by

$$
\left|\sum_{N^{\lambda}<\zeta \leq \frac{N_{2}}{k}} e\left(-\frac{\xi \zeta}{\alpha}\right)\right| \leq \min \left(N_{2},\left|\frac{\alpha}{\xi}\right|\right) \text {. }
$$

Thus using the Weil bound for Kloosterman sums we obtain

$$
\begin{aligned}
\left|\sum_{\substack{N^{\lambda}<\beta \leq \frac{N_{2}}{k} \\
(\alpha, \beta)=1}} e\left(\frac{-h(r+c q) \bar{\beta}}{\alpha k}\right)\right| \\
\quad \leq \frac{1}{\alpha} \sum_{\xi=-\left\lfloor\frac{\alpha-1}{2}\right\rfloor}\left|\sum_{\substack{\beta=0 \\
(\alpha, \beta)=1}}^{\alpha-1} e\left(\frac{\xi \beta-h \bar{\beta}(r+c q) / k}{\alpha}\right)\right| \sum_{N^{\lambda}<\zeta \leq \frac{N_{2}}{k}} e\left(-\frac{\xi \zeta}{\alpha}\right) \mid \\
\ll \alpha^{-1 / 2+\varepsilon}\left(\alpha, h \frac{r+c q}{k}\right)^{1 / 2}\left(N+\sum_{\substack{|\xi| \leq \frac{\alpha}{2} \\
\xi \neq 0}}\left|\frac{\alpha}{\xi}\right|\right) \\
\ll N^{1+2 \varepsilon} \alpha^{-1 / 2}\left(\alpha, h \frac{r+c q}{k}\right)^{1 / 2}
\end{aligned}
$$

and hence

$$
\sum_{\alpha \leq N_{1}} \alpha^{-1 / 2}\left(\alpha, h \frac{r+c q}{k}\right)^{1 / 2} \leq \sum_{d \mid h \frac{r+c q}{k}} \sqrt{d} \sum_{j \leq \frac{N_{1}}{d}}(d j)^{-1 / 2} \ll \sum_{\substack{d \mid h \frac{r+c q}{k} \\ d \leq N_{1}}} \sqrt{N_{1} / d} \ll N^{1 / 2+\varepsilon} .
$$

The contribution to $\mathcal{F}$ is

$$
\sum_{c \in \widetilde{I} \cap \mathbf{Z}} \sum_{k \mid c q+r} \sum_{1 \leq h \leq N^{\lambda}} \frac{N^{3 / 2+3 \varepsilon}}{h} \ll \frac{N^{7 / 2+5 \varepsilon}}{q} .
$$

The remaining terms are handled in a similar way. Choosing $\lambda=1 / 2$ yields the desired result. 
We need a slightly different version of the previous lemma as well

Lemma 4.4. Let $N \geq 1$ and $d_{1}, d_{2} \mid k$. Then

$$
\begin{aligned}
& \sum_{\substack{a_{i} \leq M / d_{i} \\
\left(a_{i}, q / d_{i}\right)=1}} \sum_{c \in \mathbf{Z}} \#\left\{x \in I\left(1, N d_{1} / k, a_{2}, N a_{2} d_{2}, k a_{1}, c q\right) \mid x a_{1} \equiv c q / k\left(a_{2}\right), \quad(x, q / k)=1\right\} \\
& =\frac{k \varphi(q / k)}{q} \sum_{\substack{a_{i} \leq M / d_{i} \\
\left(a_{i}, q / d_{i}\right)=1}} \sum_{\begin{array}{c}
c \in \mathbf{Z} \\
\left(a_{1}, a_{2}\right) \mid c
\end{array}} \frac{\left(a_{1}, a_{2}\right)}{a_{2}} \int_{0}^{N d_{1} / k} 1_{\left\{t \in \mathbf{R} \mid c q /\left(k a_{1}\right) \leq t \leq\left(N a_{2} d_{2}+c q\right) /\left(k a_{1}\right)\right\}}(t) d t+ \\
& O\left(\frac{N^{7 / 2+\varepsilon}}{q}\right)
\end{aligned}
$$

uniformly for $M \asymp N, q \leq N^{2-\delta}$ and $k \mid q$.

Proof. As in the proof of Lemma 4.3 we see that

$$
\begin{aligned}
& \sum_{\begin{array}{c}
a_{i} \leq M / d_{i} \\
\left(a_{i}, q / d_{i}\right)=1
\end{array}} \sum_{c \in \mathbf{Z}} \#\left\{x \in I\left(1, N d_{1} / k, a_{2}, N a_{2} d_{2}, k a_{1}, c q\right) \mid x a_{1} \equiv c q / k\left(a_{2}\right),(x, q / k)=1\right\} \\
& =\sum_{\substack{a_{i} \leq M / d_{i} \\
\left(a_{i}, q / d_{i}\right)=1}} \sum_{c \in \mathbf{Z}} \#\left\{x \in J\left(0, N d_{1} / k, 0, N a_{2} d_{2}, k a_{1}, c q\right) \mid x a_{1} \equiv c q / k\left(a_{2}\right),(x, q / k)=1\right\}+ \\
& \quad O\left(\frac{N^{3+\varepsilon}}{q}\right) .
\end{aligned}
$$

Assume $(q, l)=1$ and $l \mid r$. Then

$$
\begin{aligned}
\#\{x \in \mathbf{Z} \mid a<x \leq b, x \equiv r(q),(x, l)=1\} & =\#\{c \in \mathbf{Z} \mid(a-r) / q<c \leq(b-r) / q,(c, l)=1\} \\
& =\sum_{d \mid l} \mu(d)\left(\left[\frac{b-r}{q d}\right]-\left[\frac{a-r}{q d}\right]\right) \\
& =\sum_{d \mid l} \mu(d) \#\{x \in \mathbf{Z} \mid a<x \leq b, x \equiv r(q d)\} .
\end{aligned}
$$

Thus

$$
\begin{aligned}
& \#\left\{x \in J\left(0, N d_{1} / k, 0, N a_{2} d_{2}, k a_{1}, c q\right) \mid x a_{1} \equiv c q / k\left(a_{2}\right),(x, q / k)=1\right\}= \\
& \qquad \sum_{d \mid \frac{q}{k}} \mu(d) \#\left\{x \in J\left(0, N d_{1} / k, 0, N a_{2} d_{2}, k a_{1}, c q\right) \mid x a_{1} \equiv c q / k\left(a_{2}\right)\right\} .
\end{aligned}
$$

We can now proceed as in the proof of Lemma 4.4. The idea is the same so we only sketch the rest of the proof.

For the $\theta_{H}$ sums we are lead to consider (essentially) sums of the form

$$
\sum_{|c| \leq \frac{N^{2}}{q}} \sum_{\delta \mid \frac{q}{k}} \sum_{\substack{\alpha_{i} \leq M / d_{i} \\\left(\alpha_{i}, q / d_{i}\right)=1}} \theta_{H}\left(\frac{c q \bar{\alpha}_{1}}{k \delta \alpha_{2}}\right)
$$

and these can be estimated just as in Lemma 4.4 since (roughly speaking) the sum above just has fewer terms than the ones considered in the previous lemma (we exclude the terms that do not meet a certain coprimality condition) and all terms are non-negative. We have to be more 
careful with the exponential sum terms. We consider sums of the form

$$
\sum_{c \in I \cap \mathbf{Z}} \sum_{l \mid \frac{q}{k}} \sum_{\delta \mid c}\left|\sum_{\substack{H<\alpha_{i} \leq \frac{M}{\delta d_{i}} \\\left(\alpha_{1}, \alpha_{2}\right)=\left(\alpha_{i}, q / d_{i}\right)=1}} \sum_{1 \leq|h| \leq H} \frac{1}{h} e\left(-\frac{h c q \bar{\alpha}_{1}}{k l \delta \alpha_{2}}\right)\right|,
$$

where $I$ is an interval of length at most of order $N^{2} / q$. In the inner sum we replace the coprimality condition $\left(\alpha_{1}, q / d_{1}\right)=1$ with a Möbius sum (setting $\left.\alpha_{1}=\gamma \lambda\right)$ and get

$$
\sum_{\gamma \mid \frac{q}{d_{1}}} \mu(\gamma) \sum_{1 \leq|h| \leq H} \frac{1}{h} \sum_{\substack{H<\alpha_{2} \leq \frac{M}{\delta d_{2}} \\\left(\alpha_{2}, \gamma q / d_{2}\right)=1}} \sum_{\substack{\gamma \\ \gamma}} e\left(-\frac{\bar{\gamma} h c q \bar{\lambda}}{\left.k, \alpha_{2}\right)=1}\right) .
$$

The inner sum is estimated as in Lemma 4.3 by

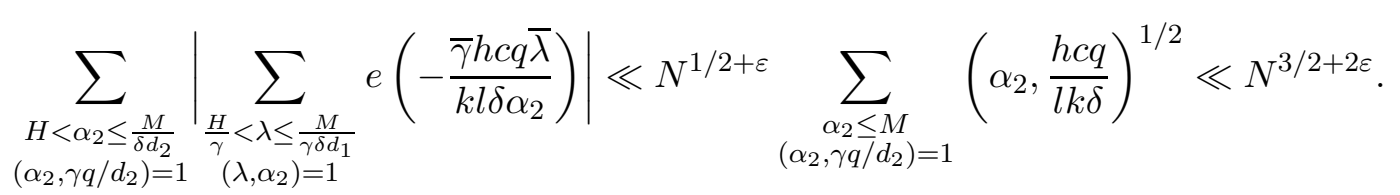

Thus we can estimate (4.2) by $\frac{N^{7 / 2+3 \varepsilon}}{q}$.

Using Lemma 4.3 we can now prove the following:

Theorem 4.5. Let $K, M, N \geq 1, q \in \mathbf{N}$ with $M \asymp N$ and $N^{\eta} \leq K$ for some $\eta>0$. Assume also that $q \leq N^{2-\delta}$ for some $\delta>0$ and $(\rho, q)=1$. Then

$$
\sum_{s=1}^{q}\left(\sum_{r \leq K} \sum_{m \equiv \rho r+s(q)} \tau_{M, N}(m)-\frac{K M N}{q}\right)^{2} \ll \frac{K^{2} N^{\max (7 / 2,4-\delta, 4-\eta)+\varepsilon}}{q}
$$

for any $\varepsilon>0$.

Proof. We may safely assume that $K \leq q$. We see that

$$
\begin{aligned}
\sum_{s=1}^{q}\left(\sum_{r \leq K} \sum_{m \equiv \rho r+s(q)} \tau_{M, N}(m)\right)^{2} & =\sum_{\substack{r \leq K \\
r^{\prime} \leq K}} \sum_{m \equiv m^{\prime}+\rho\left(r-r^{\prime}\right)(q)} \tau_{M, N}(m) \tau_{M, N}\left(m^{\prime}\right) \\
& =\sum_{|l| \leq K-1} R_{l} \sum_{m \equiv m^{\prime}+l \rho(q)} \tau_{M, N}(m) \tau_{M, N}\left(m^{\prime}\right)
\end{aligned}
$$

where $R_{l}=[K]-|l|$. We consider the innermost sum

$$
\begin{aligned}
& \sum_{m \equiv m^{\prime}+l \rho(q)} \tau_{M, N}(m) \tau_{M, N}\left(m^{\prime}\right)= \\
& \quad \#\left\{\left(a_{1}, b_{1}, a_{2}, b_{2}\right) \in \mathbf{N}^{4} \mid a_{1}, a_{2} \leq N, b_{1}, b_{2} \leq M, a_{1} b_{1} \equiv a_{2} b_{2}+\rho l(q)\right\}
\end{aligned}
$$

We want to "switch" the roles of $q$ and $a_{2}$ and apply Lemma 4.3. Note that

$$
a_{1} b_{1} \equiv a_{2} b_{2}+\rho l(q)
$$

exactly if there exists $c \in \mathbf{Z}$ such that

$$
a_{1} b_{1}-\rho l-c q=a_{2} b_{2} .
$$


Now fix $a_{1}$ and $a_{2}$. We see that (4.3) is

$$
\begin{aligned}
\sum_{\substack{a_{1}, a_{2} \leq N \\
c \in \mathbf{Z}}} \#\left\{x \in I\left(1, M, a_{2}, M a_{2}, a_{1}, \rho l+c q\right) \mid x a_{1} \equiv \rho l+c q\left(a_{2}\right)\right\} \\
=\sum_{\substack{a_{1}, a_{2} \leq N \\
c \in \mathbf{Z} \\
\left(a_{1}, a_{2}\right) \mid c q+\rho l}} \frac{\left(a_{1}, a_{2}\right)}{a_{2}} \int_{0}^{M} 1_{\left\{t \in \mathbf{R} \mid(\rho l+c q) / a_{1} \leq t \leq(\rho l+c q) / a_{1}+M a_{2} / a_{1}\right\}}(t) d t+O\left(\frac{N^{7 / 2+\varepsilon}}{q}\right) \\
=\sum_{\substack{a_{2}, a_{1} \leq N \\
\left(a_{2}, a_{1}, q\right) \mid l}} \frac{\left(a_{2}, a_{1}\right)}{a_{2}} \int_{0}^{M} \sum_{\substack{\left(t a_{1}-l \rho\right) / q-M a_{2} / q \leq c \leq\left(t a_{1}-l \rho\right) / q \\
\left(a_{2}, a_{1}\right) \mid c q+\rho l}} 1 d t+O\left(\frac{N^{7 / 2+\varepsilon}}{q}\right) \\
= \\
=\sum_{\substack{a_{2}, a_{1} \leq N \\
\left(a_{2}, a_{1}, q\right) \mid l}} \frac{\left(a_{2}, a_{1}\right)}{a_{2}} \frac{M a_{2}\left(a_{2}, a_{1}, q\right)}{q\left(a_{2}, a_{1}\right)}+O\left(N^{2+\varepsilon}+\frac{N^{7 / 2+\varepsilon}}{q}\right) \\
=\frac{M^{2}}{q} \sum_{\substack{a_{2}, a_{1} \leq N \\
\left(a_{2}, a_{1}, q\right) \mid l}}\left(a_{2}, a_{1}, q\right)+O\left(\frac{N^{\max (7 / 2,4-\delta)+\varepsilon}}{q}\right) .
\end{aligned}
$$

Again we consider the entire sum and see (using Lemma 3.1)

$$
\begin{aligned}
\sum_{|l| \leq K} R_{l} \sum_{\substack{a_{2}, a_{1} \leq N \\
\left(a_{2}, a_{1}, q\right) \mid l}}\left(a_{2}, a_{1}, q\right)= & \sum_{a_{1} \leq N} \sum_{|l| \leq K} R_{l} \sum_{\substack{a_{2} \leq N \\
\left(a_{2}, a_{1}, q\right) \mid l}}\left(a_{2}, a_{1}, q\right) \\
= & 2[K] \sum_{a_{1}=1}^{N}\left(K N+O\left((K+N) q^{\varepsilon}\right)\right)+O\left(K N^{2} q^{\varepsilon}\right)- \\
& \sum_{a_{1}=1}^{N}\left(K^{2} N+O\left(K(K+N) q^{\varepsilon}\right)\right) \\
= & K^{2} N^{2}+O\left(N K(N+K) q^{\varepsilon}\right) .
\end{aligned}
$$

Thus

$$
\begin{aligned}
\sum_{s=1}^{q}\left(\sum_{r \leq K} \sum_{m \equiv \rho r+s(q)} \tau_{M, N}(m)-\frac{K M N}{q}\right)^{2} & =\sum_{\substack{r \leq K \\
r^{\prime} \leq K}} \sum_{m \equiv m^{\prime}+\rho\left(r-r^{\prime}\right)(q)} \tau_{M, N}(m) \tau_{M, N}\left(m^{\prime}\right)- \\
& \frac{K^{2} M^{2} N^{2}}{q}+O\left(\frac{K N^{4}+K^{2} N^{3}}{q}\right) \\
= & O\left(\frac{K^{2} N^{\max (7 / 2,4-\delta, 4-\eta)+\varepsilon}}{q}\right) .
\end{aligned}
$$

This also shows that for the individual terms the asymptotics one should expect from Conjecture 1.2 holds for a subset of $\{s \in \mathbf{N} \mid 1 \leq s \leq q\}$ of full density in the following sense:

Corollary 4.6. Let $\nu>0$ and assumptions be as in Theorem 4.5. Then

$$
\#\left\{s \in\{1, \ldots, q\}|| 1-\frac{q}{K M N} \sum_{r \leq K} \sum_{m \equiv \rho r+s(q)} \tau_{M, N}(m) \mid>\nu\right\} \ll q N^{-\min (\delta, 1 / 2, \eta)+\varepsilon}
$$

uniformly in $K, q$ and $\rho$ for any $\varepsilon>0$. 
In this connection it should be mentioned that if $s$ is "bad" then so is its neighbors. In fact we see that just one bad $s$ (in the sense of Corollary 4.6) will imply that there are at least $K^{1-\varepsilon}$ bad values of $s$.

We now proceed to the proof of Theorem 1.8 ,

Proof of Theorem 1.8. Recall that $\varphi(q) / q \gg q^{-\varepsilon}$ for any $\varepsilon>0$. Applying (3.1) we see that

$$
\begin{aligned}
\sum_{(r, q)=k} \sum_{m \equiv r(q)} \tau_{M, N}(m) & =\sum_{(m, q)=k} \tau_{M, N}(m) \\
& =\sum_{c \mid k} \varphi(M / c, q / c) \varphi(N c / k, q / k) \\
& =\sum_{c \mid k} \frac{\varphi(q / c) \varphi(q / k) c M N}{q^{2}}+O\left(q^{\varepsilon} N\right) .
\end{aligned}
$$

We proceed as in the proof of Theorem 4.5 and obtain using Lemma 4.4

$$
\begin{aligned}
& \sum_{(q, r)=k} \sum_{\substack{m \equiv r(q) \\
m^{\prime} \equiv r(q)}} \tau_{M, N}(m) \tau_{M, N}\left(m^{\prime}\right) \\
& =\sum_{d_{1}, d_{2} \mid k} \sum_{\substack{\alpha_{1} \leq \frac{M}{d_{1}} \\
\left(\alpha_{1}, q / d_{1}\right)=1}} \sum_{\substack{\alpha_{2} \leq \frac{M}{d_{2}} \\
\left(\alpha_{2}, q / d_{2}\right)=1}} \sum_{\substack{c \in \mathbf{Z} \\
\left(\alpha_{1}, \alpha_{2}\right) \mid c}} \\
& \#\left\{x \in I\left(1, \frac{N d_{1}}{k}, \alpha_{2}, N \alpha_{2} d_{2}, k \alpha_{1}, c q\right) \mid x \alpha_{1} \equiv \frac{c q}{k}\left(\alpha_{2}\right),\left(x, \frac{q}{k}\right)=1\right\} \\
& =\sum_{d_{1}, d_{2} \mid k} \sum_{\substack{\alpha_{1} \leq \frac{M}{d_{1}} \\
\left(\alpha_{1}, q / d_{1}\right)=1}} \sum_{\substack{\alpha_{2} \leq \frac{M}{d_{2}} \\
\left(\alpha_{2}, q / d_{2}\right)=1}} \sum_{\substack{c \in \mathbf{Z} \\
\left(\alpha_{1}, \alpha_{2}\right) \mid c}} \frac{\left(\alpha_{1}, \alpha_{2}\right) \varphi(q / k) k}{\alpha_{2} q} \times \\
& \int_{0}^{\frac{N d_{1}}{k}} 1_{\left\{t \in \mathbf{R} \mid c q /\left(k \alpha_{1}\right)<t \leq\left(c q+N \alpha_{2} d_{2}\right) /\left(k \alpha_{1}\right)\right\}}(t) d t+O\left(\frac{N^{7 / 2+\varepsilon}}{q}\right) \\
& =\frac{N^{2} \varphi(q / k)}{q^{2}} \sum_{i=1,2} \sum_{d_{i} \mid k} d_{1} d_{2} \sum_{\substack{\alpha_{1} \leq \frac{M}{d_{1}} \\
\left(\alpha_{1}, q / d_{1}\right)=1}} \sum_{\substack{\alpha_{2} \leq \frac{M}{d_{2}} \\
\left(\alpha_{2}, q / d_{2}\right)=1}} 1+O\left(\frac{N^{\max (4-\delta, 7 / 2)+\varepsilon}}{q}\right) \\
& =\frac{M^{2} N^{2} \varphi(q / k)}{q^{2}}\left(\sum_{d \mid k} d \varphi(q / d)\right)^{2}+O\left(\frac{N^{\max (4-\delta, 7 / 2)+\varepsilon}}{q}\right) .
\end{aligned}
$$

This proves the theorem.

Finally we prove Theorem 1.9 ,

Proof of Theorem 1.9. We see that

$$
\begin{aligned}
\sum_{(\rho, q)=1} \sum_{r \leq K} \sum_{m \equiv \rho r(q)} \tau_{M, N}(m) & =\sum_{r \leq K} \frac{\varphi(q)}{\varphi(q /(q, r))} \sum_{(m, q)=(r, q)} \tau_{M, N}(m) \\
& =\sum_{\substack{k \leq K \\
k \mid q}} \frac{\varphi(q)}{\varphi(q / k)} \#\{r \in \mathbf{N} \mid r \leq K,(r, q)=k\} \times \\
& \#\left\{(a, b) \in \mathbf{N}^{2} \mid a \leq M, b \leq N,(a b, q)=k\right\} .
\end{aligned}
$$


In the same way we see that

$$
\begin{aligned}
\#\left\{(a, b) \in \mathbf{N}^{2} \mid a \leq M, b \leq\right. & N,(a b, q)=k\} \\
& =\sum_{l \mid k} \#\{b \in \mathbf{N} \mid b \leq N,(b, q)=l\} \times \\
& \#\{a \in \mathbf{N} \mid a \leq M,(a, q / l)=k / l\} \\
& =\sum_{l \mid k}\left(\frac{N}{q} \varphi(q / l)+O\left(q^{\varepsilon}\right)\right)\left(\frac{M l}{q} \varphi(q / k)+O\left(q^{\varepsilon}\right)\right) \\
& =\frac{\varphi(q / k) M N}{q^{2}} \sum_{l \mid k} l \varphi(q / l)+O\left(N q^{\varepsilon}\right) \\
& \leq \frac{M N \tau(k)}{k}+O\left(N q^{\varepsilon}\right) .
\end{aligned}
$$

Thus

$$
\begin{aligned}
\sum_{\substack{k \leq K \\
k \mid q}} \#\{ & \left.(a, b) \in \mathbf{N}^{2} \mid a \leq M, b \leq N,(a b, q)=k\right\} \\
& =M N-\sum_{\substack{k>K \\
k \mid q}} \#\left\{(a, b) \in \mathbf{N}^{2} \mid a \leq M, b \leq N,(a b, q)=k\right\} \\
& =M N+O\left(\frac{N^{2} q^{\varepsilon}}{K}+N q^{\varepsilon}\right) .
\end{aligned}
$$

Combining (3.2) and (4.5) it follows that

$$
\sum_{(\rho, q)=1} \sum_{r \leq K} \sum_{m \equiv \rho r(q)} \tau_{M, N}(m)=\frac{\varphi(q) K M N}{q}+O\left(N^{2} q^{\varepsilon}+K N q^{\varepsilon}\right) .
$$

Now we look at

$$
\left(\sum_{r \leq K} \sum_{m \equiv r \rho(q)} \tau_{M, N}(m)-\frac{K M N}{q}\right)^{2}
$$

and rewrite it as

$$
\begin{aligned}
& \left(\sum_{r \leq K}\left(\sum_{m \equiv r \rho(q)} \tau_{M, N}(m)-\frac{M N}{q^{2}} \sum_{d \mid(r, q)} d \varphi\left(\frac{q}{d}\right)\right)\right)^{2}+\left(\frac{M N}{q^{2}}\left(K q-\sum_{r \leq K} \sum_{d \mid(r, q)} d \varphi\left(\frac{q}{d}\right)\right)\right)^{2}- \\
& 2\left(\sum_{r \leq K}\left(\sum_{m \equiv r \rho(q)} \tau_{M, N}(m)-\frac{M N}{q^{2}} \sum_{d \mid(r, q)} d \varphi\left(\frac{q}{d}\right)\right)\right)\left(\frac{M N}{q^{2}}\left(K q-\sum_{r \leq K} \sum_{d \mid(r, q)} d \varphi\left(\frac{q}{d}\right)\right)\right) .
\end{aligned}
$$

Lemma 3.3 implies that

$$
\left|\frac{M N}{q^{2}}\left(K q-\sum_{r \leq K} \sum_{d \mid(r, q)} d \varphi\left(\frac{q}{d}\right)\right)\right| \ll \frac{N^{2}(K+q) q^{\varepsilon}}{q^{2}} .
$$

We also see that Lemma 3.3 and (4.6) implies

$$
\left|\sum_{(\rho, q)=1} \sum_{r \leq K}\left(\sum_{m \equiv r \rho(q)} \tau_{M, N}(m)-\frac{M N}{q^{2}} \sum_{d \mid(r, q)} d \varphi\left(\frac{q}{d}\right)\right)\right| \ll K N^{2} q^{\varepsilon}\left(\frac{1}{K}+\frac{1}{q}+\frac{1}{N}\right) .
$$


Thus it remains to look at

$$
\sum_{(\rho, q)=1}\left(\sum_{r \leq K}\left(\sum_{m \equiv r \rho(q)} \tau_{M, N}(m)-\frac{M N}{q^{2}} \sum_{d \mid(r, q)} d \varphi\left(\frac{q}{d}\right)\right)\right)^{2} .
$$

Using Cauchy-Schwarz inequality and Theorem 1.8 we estimate (4.9) by

$$
\begin{aligned}
K \sum_{r \leq K} & \sum_{(\rho, q)=1}\left(\sum_{m \equiv r \rho(q)} \tau_{M, N}(m)-\frac{M N}{q^{2}} \sum_{d \mid(r, q)} d \varphi\left(\frac{q}{d}\right)\right)^{2} \\
& =K \sum_{r \leq K} \frac{\varphi(q)}{\varphi(q /(q, r))} \sum_{(s, q)=(r, q)}\left(\sum_{m \equiv s(q)} \tau_{M, N}(m)-\frac{M N}{q^{2}} \sum_{d \mid(r, q)} d \varphi\left(\frac{q}{d}\right)\right)^{2} \\
& \ll \frac{K N^{\max (7 / 2,4-\delta)+\varepsilon}}{q} \sum_{r \leq K} \frac{\varphi(q)}{\varphi(q /(q, r))} \\
& \ll \frac{K N^{\max (7 / 2,4-\delta)+\varepsilon}}{q} \sum_{r \leq K}(q, r) \\
& \ll \frac{K^{2} N^{\max (7 / 2,4-\delta)+2 \varepsilon}}{q}
\end{aligned}
$$

Together with the estimates (4.7) and (4.8) this proves the theorem.

\section{Estimates FOR $\tau_{M}^{*}$ AND $\tau_{M}$}

Through out this section we will restrict our discussion to $\tau_{M}^{*}$ though the results (with suitable modifications) clearly can be extended to cover $\tau_{M, N}$ as well.

We see that

$$
\sum_{m \leq x} \tau_{M}(m)=\sum_{m \leq x} \sum_{d=1}^{[M]} \delta_{d}(m)=\sum_{d=1}^{[M]} \frac{x}{d}+O(M)=x \log M+O(M+x) .
$$

Let $\tau$ denote the usual divisor function and note that

$$
\tau_{M}^{*}(m)= \begin{cases}\tau(m) & \text { if } m \leq M \\ 2 \tau_{M}(m)-\tau(m) & \text { if } M<m<M^{2} \\ 0 & \text { if } m \geq M^{2}\end{cases}
$$

It is well known that

$$
\sum_{m \leq x} \tau(m)=x \log x+(2 \gamma-1) x+O(\sqrt{x})
$$

Better estimates for the error term are known but that is irrelevant for our application. Thus

$$
\sum_{m \leq x} \tau_{M}^{*}(m)= \begin{cases}x \log x+(2 \gamma-1) x+O(\sqrt{x}) & \text { if } x \leq M \\ x \log \frac{M^{2}}{x}+O(x) & \text { if } M<x<M^{2}, \\ {[M]^{2}} & \text { if } x \geq M^{2}\end{cases}
$$

where the constants implied are absolute.

Conjecture 1.5 is probably hard to prove. We can however, give an estimate for the sum using a result due to Nair and Tenenbaum [11. Before we state the result we need to introduce some notation. Let $F: \mathbf{N} \rightarrow \mathbf{R}_{+}$. We say that $F \in \mathcal{M}(A, B, \varepsilon)$ if $F$ satisfies (for $(m, n)=1$ )

$$
F(m n) \leq \min \left(A^{\Omega(m)}, B m^{\varepsilon}\right) F(n)
$$


for some $A, B \geq 0$, where $\Omega(m)$ denotes the total number of prime factors of $m$, counted with multiplicity. The following theorem is an immediate consequence of [11, Theorem 1]:

Theorem 5.1. Let $F \in \mathcal{M}\left(A, B, \frac{\varepsilon \delta}{3}\right), 0<\delta \leq 1,0<\varepsilon<\frac{1}{8}$. Then

$$
\sum_{\substack{x<n \leq x+y \\ n \equiv r(q)}} F(n) \ll \frac{y}{\varphi(q) \log x} \sum_{\substack{n \leq \frac{x}{q} \\(n, q)=1}} \frac{F(n)}{n}
$$

uniformly for $(r, q)=1, x^{\frac{1+4 \varepsilon \delta}{1+\delta}} \leq y \leq x, x \geq c_{0} q^{1+\delta}$, where $c_{0}$ and the constant implied depends at most on $A, B, \delta$ and $\varepsilon$.

Proposition 1.6 can be proved quite easily, since $\tau_{N}^{*}$ is closely related to the Hooley $\Delta$-function defined by

$$
\Delta(n)=\max _{u \in \mathbf{R}} \#\left\{d \in \mathbf{N}\left|e^{u}<d \leq e^{u+1}, d\right| n\right\} .
$$

We see that for all $N \geq 1, k \in \mathbf{N}$ we have

$$
\tau_{N}^{*}(m) \leq 2 k \Delta(m)
$$

whenever $\frac{N^{2}}{2^{k}}<m \leq \frac{N^{2}}{2^{k-1}}$. One easily checks that $\Delta \in \mathcal{M}(2, B, \varepsilon)$ for any $\varepsilon>0$ and suitable $B$ (chosen according to $\varepsilon$ ). In [15] the following estimate was proved

$$
\sum_{n \leq x} \frac{\Delta(n)}{n} \ll e^{\sqrt{(2+\varepsilon)(\log \log x)(\log \log \log x)}} \log x .
$$

Using Theorem 5.1 this implies that

$$
\sum_{\substack{m \equiv r(q) \\ N^{2} / 2^{k}<m \leq N^{2} / 2^{k-1}}} \Delta(m) \ll \frac{N^{2}}{2^{k} \varphi(q)} e^{\sqrt{(2+\varepsilon)(\log \log N)(\log \log \log N)}} .
$$

Now choose $l$ such that $c_{0} q^{1+\delta}<N^{2} / 2^{l}<N^{2-\delta^{\prime}}$ for some fixed $\delta^{\prime}>0$. Then

$$
\sum_{m \equiv r(q)} \tau_{N}^{*}(m) \leq \sum_{\substack{m \equiv r(q) \\ m \leq N^{2} / 2^{l}}} \tau(m)+2 \sum_{k=1}^{l} k \sum_{\substack{m \equiv r(q) \\ N / 2^{k}<m \leq N^{2} / 2^{k-1}}} \Delta(m) .
$$

From (5.4) and the Linnik-Vinogradov estimate (1.6) it follows that

$$
\sum_{m \equiv r(q)} \tau_{N}^{*}(m) \ll \frac{N^{2}}{q}+\frac{N^{2} e^{\sqrt{(2+\varepsilon)(\log \log N)(\log \log \log N)}}}{\varphi(q)} \sum_{k=1}^{\infty} \frac{k}{2^{k}} .
$$

This proves Proposition 1.6.

Note that for $x \gg 1$

$$
e^{\sqrt{(2+\varepsilon)(\log \log x)(\log \log \log x)}} \ll(\log x)^{\varepsilon^{\prime}}
$$

for any $\varepsilon^{\prime}>0$. To see this note that

as $x \rightarrow \infty$. In particular

$$
\frac{\log \log \log x}{\log \log x} \rightarrow 0
$$

$$
\frac{\log \log \log x}{\log \log x} \leq \frac{\varepsilon^{\prime 2}}{2+\varepsilon}
$$

for any $\varepsilon^{\prime}>0$ for $x$ large. Hence

$$
(2+\varepsilon)(\log \log x)(\log \log \log x) \leq\left(\varepsilon^{\prime} \log \log x\right)^{2} .
$$


From this (5.5) follows easily.

To prove Theorem 1.7 we need the following lemma.

Lemma 5.2. Let $\delta, \varepsilon>0$. Let $q \leq x^{1-\delta}$ and $2 \leq N$. Assume also that $N \geq q^{\varepsilon}$. Then

$$
\sum_{\substack{n \leq x \\(n, q)=1}} \frac{\tau_{N}(n)}{n} \ll \frac{\varphi(q)^{2} \log x \log N}{q^{2}} .
$$

Proof. We see that

$$
\sum_{\substack{n \leq x \\(n, q)=1}} \frac{\tau_{N}(n)}{n}=\sum_{\substack{a b \leq x, a \leq N \\(a, q)=(b, q)=1}} \frac{1}{a b}=\sum_{\substack{a \leq N \\(a, q)=1}} \frac{1}{a} \sum_{\substack{b \leq x / a \\(b, q)=1}} \frac{1}{b}
$$

Thus we must consider

$$
\begin{aligned}
\sum_{\substack{a \leq Y \\
(a, q)=1}} \frac{1}{a} & =\sum_{a \leq Y} \frac{1}{a} \sum_{\substack{d|q \\
d| a}} \mu(d) \\
& =\sum_{d \mid q} \frac{\mu(d)}{d} \sum_{\substack{\alpha \leq Y / d \\
\frac{1}{\alpha}}} \\
& =\sum_{d \mid q} \frac{\mu(d)}{d}\left(\log \frac{Y}{d}+\gamma+O\left(\frac{d}{Y}\right)\right) \\
& =(\log Y+\gamma) \frac{\varphi(q)}{q}+O\left(\frac{\tau(q)}{Y}\right)-\sum_{d \mid q} \frac{\mu(d) \log d}{d}
\end{aligned}
$$

Let $q^{\prime}$ denote the square free part of $q$ and $p$ be a prime number. We see that

$$
\begin{aligned}
\sum_{d \mid q} \frac{\mu(d) \log d}{d} & =\sum_{d \mid q} \frac{\mu(d)}{d} \sum_{c \mid d} \Lambda(c) \\
& =\sum_{c \mid q^{\prime}} \Lambda(c) \sum_{\substack{c|d \\
d| q^{\prime}}} \frac{\mu(d)}{d} \\
& =\sum_{p \mid q^{\prime}} \Lambda(p) \sum_{\delta \mid \frac{q^{\prime}}{p}} \frac{\mu(\delta p)}{\delta p} \\
& =-\sum_{p \mid q^{\prime}} \frac{\Lambda(p)}{p} \sum_{\delta \mid \frac{q^{\prime}}{p}} \frac{\mu(\delta)}{\delta}
\end{aligned}
$$

Thus

$$
\sum_{d \mid q} \frac{\mu(d) \log d}{d}=O\left(\sum_{p \mid q^{\prime}} \frac{\Lambda(p)}{p}\right)=O\left(\sum_{p \mid q} \frac{\log p}{p}\right) .
$$

We split the last sum in two parts:

$$
\sum_{p \mid q} \frac{\log p}{p}=\sum_{\substack{p \mid q \\ p \leq(\log q)^{2}}} \frac{\log p}{p}+\sum_{\substack{p \mid q \\ p>(\log q)^{2}}} \frac{\log p}{p} .
$$


Clearly

$$
\sum_{\substack{p \mid q \\ p>(\log q)^{2}}} \frac{\log p}{p} \leq(\log q)^{-2} \sum_{p \mid q} \log p \leq 1 .
$$

We know that

$$
\sum_{p \leq x} \frac{1}{p}=O(\log \log x)
$$

Hence

$$
\sum_{\substack{p \mid q \\ p \leq(\log q)^{2}}} \frac{\log p}{p} \leq 2 \log \log q \sum_{p \leq q} p^{-1}=O\left((\log \log q)^{2}\right) .
$$

From this it follows that

$$
\sum_{\substack{a \leq Y \\(a, q)=1}} \frac{1}{a}=(\log Y+\gamma) \frac{\varphi(q)}{q}+O\left(\frac{\tau(q)}{Y}\right)+O\left((\log \log q)^{2}\right) .
$$

Recall that $\varphi(q) \gg \frac{q}{\log \log q}$. Thus it follows that

$$
\sum_{\substack{a \leq N \\(a, q)=1}} \frac{1}{a} \ll \frac{\varphi(q) \log N}{q}
$$

and

$$
\sum_{\substack{b \leq x \\(b, q)=1}} \frac{1}{b} \ll \frac{\varphi(q) \log x}{q} .
$$

The result now follows from (5.6).

Theorem 1.7 follows immediately from Theorem 5.1 and Lemma 5.2 since $\tau_{N} \in \mathcal{M}(2, B, \varepsilon)$.

\section{Proof of Theorem 1.4}

We follow Section 5 in $[5]$. For $\delta \in(0,1)$ define

$$
\mathcal{R}(M, \alpha, \delta)=\#\left\{(x, y) \in \mathbf{Z}^{2}|| x \alpha-y|\leq \delta,| x \mid \leq M\right\} .
$$

The proof of Theorem 1.4 is based on the following identity

$$
\mathcal{S}(M, N, K, p, q)=\sum_{a \leq N} \mathcal{R}(M, a p / q, K / q)=N+2 \sum_{|r| \leq K} \sum_{p m \equiv r(q)} \tau_{M, N}(m) .
$$

We can transform it into a lattice point problem since

$$
\left\{(x, y) \in \mathbf{Z}^{2}|| x \alpha-y|\leq \delta,| x \mid \leq M\right\}=\left\{(x, y) \in \mathbf{Z}^{2} \mid x \mathbf{u}+y \mathbf{v} \in[-\sqrt{M \delta}, \sqrt{M \delta}]^{2}\right\}
$$

where $\mathbf{u}=(\sqrt{\delta / M}, \alpha \sqrt{M / \delta})$ and $\mathbf{v}=(0,-\sqrt{M / \delta})$. Since $\mathbf{u}$ and $\mathbf{v}$ generate a lattice of determinant 1 we obtain

$$
\mathcal{R}(M, \alpha, \delta)=4 M \delta+O\left(\sqrt{M \delta} / \lambda_{1}\right)+O(1)
$$

where $\lambda_{1}$ is the length of the shortest non-zero vector in the lattice. We have $\delta=K / q$ and $\alpha=a p / q$ in our case. Thus we expect that the main term in $\mathcal{S}(M, N, K, p, q)$ is $K M N / q$. It is the error term $O\left(\sqrt{M \delta} / \lambda_{1}\right)$ that needs attention. In particular one is concerned with the case where $\lambda_{1}$ is small. The idea is to consider $\sqrt{M \delta} / \lambda_{1}$ in dyadic intervals

$$
E<\sqrt{M \delta} / \lambda_{1} \leq 2 E
$$


Note that $E$ can be at most $M$ since $\lambda_{1} \geq \sqrt{\delta / M}$. The $a$ 's for which $E \leq \sqrt{M K / q}$ contribute $N \sqrt{K M / q}$ to the error term for $\mathcal{S}(M, N, K, p, q)$. Following Lemma 4 in 5 the contribution from values of $a$ for which $E \geq \sqrt{M K / q}$ can be estimated by

$$
\sum_{\sqrt{M K / q} \leq E=2^{k} \leq M} \sum_{1 \leq F=2^{h} \leq N} \operatorname{EFV}(M / E, N / F, K /(q E F), p / q),
$$

where

$$
V(A, B, D, \beta)=\#\left\{(a, b, z) \in \mathbf{N}^{2} \times \mathbf{Z}|a \leq A, b \leq B,(a b, z)=1,| a b \beta-z \mid \leq D\right\} .
$$

Using Lemma 6 and Lemma 7 in [5] we obtain the estimate

$$
\sum_{\sqrt{M K / q} \leq E=2^{k} \leq M} \sum_{\substack{1 \leq F=2^{h} \leq N \\ E F \leq(\log N)^{5 / 4}}} E F V(M / E, N / F, K /(q E F), p / q) \ll N(K N / q)^{7 / 8} .
$$

Clearly we have

$$
V(A, B, D, p / q) \leq \sum_{|r| \leq q D} \sum_{\begin{array}{c}
m \leq A B \\
p m \equiv r(q)
\end{array}} \tau_{A, B}(m) \leq \sum_{|r| \leq q D} \sum_{\substack{m \leq A B \\
p m \equiv r(q)}} \tau(m) .
$$

The previous results in the present paper suggest that there is a loss of roughly a factor $\log (A B)$ in the last inequality, but the last estimate will be sufficient for our purpose.

We now need the fact that $p / q$ is of type $(2+\gamma, \mathcal{K})$. This implies

$$
\left|\frac{p}{q}-\frac{z}{x y}\right| \geq \frac{1}{\mathcal{K}(x y)^{2+\gamma}}
$$

unless $x y \mid q$ (remember that $(x y, z)=1)$. Thus if

$$
\left|\frac{x y p}{q}-z\right| \leq \frac{K}{q E F}
$$

we conclude that

$$
(x y)^{1+\gamma} \geq \frac{q E F \mathcal{K}}{K}
$$

unless $x y \mid q$. Thus for such $x y$ we can assume that

$$
E F \leq\left(\frac{(M N)^{1+\gamma} K}{q \mathcal{K}}\right)^{\frac{1}{2+\gamma}}
$$

Using the assumption (1.2) we see that

$$
\frac{M N}{E F} \geq \frac{M N}{\left(\frac{(M N)^{1+\gamma} K}{q \mathcal{K}}\right)^{\frac{1}{2+\gamma}}} \gg q^{1+\frac{\delta(1+\gamma)}{2+\gamma}} .
$$

It follows that

$$
V(M / E, N / F, K /(q E F), p / q) \leq \sum_{|r| \leq \frac{K}{E F}} \sum_{\substack{m \leq \frac{M N}{E F} \\ p m \equiv r(q)}} \tau(m)+\tau(q)^{2} .
$$

Using the Linnik-Vinogradov estimate (1.6) one easily deduces that for $q \ll x^{1-\delta}$

$$
\sum_{|r| \leq S} \sum_{\substack{m \leq x \\ m \equiv s(q)}} \tau(m) \ll\left(\tau(q)^{2}+S(\log \log q)^{2}\right) \frac{x \log x}{q},
$$


where the constant implied depends on $\delta$ only. Since (6.1) holds we can use this result and we obtain

$$
V(M / E, N / F, K /(q E F), p / q) \ll\left(\tau(q)^{2}+\frac{K}{E F}(\log \log q)^{2}\right) \frac{N^{2} \log N}{E F q} .
$$

Clearly

$$
\sum_{\sqrt{M K / q} \leq E=2^{k} \leq M} \sum_{\substack{1 \leq F=2^{h} \leq N \\ E F \geq(\log N)^{5 / 4}}} 1 \ll(\log N)^{2} .
$$

For $t \in(0,1)$ we recall the formula

$$
\sum_{j=0}^{n}(j+1) t^{j}=\frac{(t-1)(n+1) t^{n}+1-t^{n+1}}{(1-t)^{2}} .
$$

Using this we see that

$$
\sum_{1 \leq E=2^{k} \leq M} \sum_{\substack{1 \leq F=2^{h} \leq N \\ E F \geq(\log N)^{5 / 4}}} \frac{1}{E F} \ll \sum_{\frac{5}{4} \log \log N \leq l \leq 3 \log N} \frac{l+1}{2^{l}} \ll \frac{\log \log N}{(\log N)^{5 / 4}} .
$$

This implies

$$
\begin{aligned}
\sum_{\sqrt{M K / q} \leq E=2^{k} \leq M} \sum_{\substack{1 \leq F=2^{h} \leq N \\
E F \geq(\log N)^{5 / 4}}} E F V(M / E, N / F, K /(q E F), p / q) \\
\ll \frac{N^{2}}{q}\left(\tau(q)^{2}(\log N)^{3}+\frac{K(\log \log N)^{2}}{(\log N)^{1 / 4}}\right) .
\end{aligned}
$$

\section{REFERENCES}

[1] W. D. Banks, D. R. Heath-Brown and I. E. Shparlinski. On the average value of divisor sums in arithmetic progressions. Int. Math. Res. Not. (2005), no. 1, 1-25.

[2] M. V. Berry and M. Tabor. Level clustering in the regular spectrum. Proc. Roy. Soc. London A 356 (1977), 375-394.

[3] V. Blomer. The average value of divisor sums in arithmetic progressions. Quart. J. Math. 59 (2008), 275-286.

[4] J. B. Friedlander and H. Iwaniec. Incomplete Kloosterman sums and a divisor problem. Ann. of Math. 121 (1985), no. 2, 319-350.

[5] D. R. Heath-Brown. Pair correlation for fractional parts of $\alpha n^{2}$. arXiv e-Print: math.NT/0904.0714v1.

[6] D. R. Heath-Brown. The fourth power moment of the Riemann zeta function. Proc. London Math. Soc. 33 (1979), 385-422.

[7] H. Iwaniec and E. Kowalski. Analytic number theory. AMS (2004).

[8] Y. V. Linnik and A. I. Vinogradov. Estimate of the sum of the number of divisors in a short segment of an arithmetic progression. Uspehi Mat. Nauk 12 (1957), no. 4 (76), 277-280.

[9] J. Marklof and A. Strömbergsson. Equidistribution of Kronecker sequences along closed horocycles. Geom. Funct. Anal. 13 (2003), no. 6, 1239-1280.

[10] S. J. Miller and R. Takloo-Bighash. An invitation to modern number theory. Princeton University Press (2006).

[11] M. Nair and G. Tenenbaum. Short sums of certain arithmetic functions. Acta Math. 180 (1998), 119-144.

[12] Z. Rudnick and P. Sarnak. The pair correlation function of fractional parts of polynomials. Comm. Math. Phys. 194 (1998), no. 1, 61-70.

[13] Z. Rudnick, P. Sarnak and A. Zaharescu. The distribution of spacings between the fractional parts of $n^{2} \alpha$. Invent. Math. 145 (2001), no. 1, 37-57.

[14] P. Shiu. A Brun-Titschmarsh theorem for multiplicative functions. J. Reine Angew. Math. 313 (1980), 161170.

[15] G. Tenenbaum. Sur une question d'Erdös et Schinzel, II. Invent. Math. 99 (1990), 215-224.

[16] H. Weyl. Über die Gleichverteilung von Zahlen mod. Eins. Math. Ann. 77 (1916), 313-352.

[17] A. Zaharescu. Correlation of fractional parts of $n^{2} \alpha$. Forum Math. 15 (2003), 1-21. 
Department of Pure Mathematics and Mathematical Statistics, University of Cambridge, WilBerforce Road, Cambridge CB3 0WB, United Kingdom

E-mail address: lee@imf.au.dk 